\title{
THE CLUSTERING OF EXTREMELY RED OBJECTS
}

\author{
David P. Palamara ${ }^{1,2}$, Michael J. I. Brown ${ }^{1,2}$, Buell T. Jannuzi ${ }^{3,4}$, Arjun Dey ${ }^{3}$, Daniel Stern $^{5}$, Kevin A. Pimbblet ${ }^{1,2}$, \\ Benjamin J. Weiner ${ }^{4}$, Matthew L. N. Ashby ${ }^{6}$, C. S. KochaneK ${ }^{7,8}$, Anthony Gonzalez ${ }^{9}$, Mark Brodwin $^{10}$, \\ EMERIC Le Floc' ${ }^{11}$, AND MARCIA RIEKE ${ }^{4}$ \\ ${ }^{1}$ School of Physics, Monash University, Clayton, Victoria 3800, Australia \\ ${ }^{2}$ Monash Center for Astrophysics (MoCA), Monash University, Clayton, Victoria 3800, Australia \\ ${ }^{3}$ National Optical Astronomy Observatory, 950 North Cherry Avenue, Tucson, AZ 85719, USA \\ ${ }^{4}$ Steward Observatory, University of Arizona, 933 North Cherry Avenue, Tucson, AZ, 85721, USA \\ 5 Jet Propulsion Laboratory, California Institute of Technology, Pasadena, CA 91109, USA \\ ${ }^{6}$ Harvard-Smithsonian Center for Astrophysics, 60 Garden Street, Cambridge, MA 02138, USA \\ ${ }^{7}$ Department of Astronomy, The Ohio State University, 140, West 18th Avenue, Columbus, OH 43210, USA \\ ${ }^{8}$ Center for Cosmology and Astroparticle Physics, The Ohio State University, 191 West Woodruff Avenue, Columbus, OH 43210, USA \\ ${ }^{9}$ Department of Astronomy, University of Florida, Gainesville, FL 32611-2055, USA \\ ${ }^{10}$ Department of Physics and Astronomy, University of Missouri, 5110 Rockhill Road, Kansas City, MO 64110, USA \\ ${ }^{11}$ Laboratoire AIM, CEA-Saclay-CNRS-Universite Paris Diderot, Service d'Astrophysique, Orme des Merisiers, F-91191 Gif-sur-Yvette, France \\ Received 2012 June 18; accepted 2012 November 27; published 2013 January 23
}

\begin{abstract}
We measure the clustering of extremely red objects $(E R O s)$ in $\approx 8 \mathrm{deg}^{2}$ of the NOAO Deep Wide Field Survey Boötes field in order to establish robust links between $\operatorname{ERO}(z \approx 1.2)$ and local galaxy $(z<0.1)$ populations. Three different color selection criteria from the literature are analyzed to assess the consequences of using different criteria for selecting EROs. Specifically, our samples are $\left(R-K_{s}\right)>5.0(28,724$ galaxies $),\left(I-K_{s}\right)>4.0$ (22,451 galaxies), and $(I-[3.6])>5.0(64,370$ galaxies $)$. Magnitude-limited samples show the correlation length $\left(r_{0}\right)$ to increase for more luminous EROs, implying a correlation with stellar mass. We can separate star-forming and passive ERO populations using the $\left(K_{s}-[24]\right)$ and ([3.6] $\left.-[24]\right)$ colors to $K_{s}=18.4$ and [3.6] $=17.5$, respectively. Star-forming and passive EROs in magnitude-limited samples have different clustering properties and host dark halo masses and cannot be simply understood as a single population. Based on the clustering, we find that bright passive EROs are the likely progenitors of $\gtrsim 4 L^{*}$ elliptical galaxies. Bright EROs with ongoing star formation were found to occupy denser environments than star-forming galaxies in the local universe, making these the likely progenitors of $\gtrsim L^{*}$ local ellipticals. This suggests that the progenitors of massive $\gtrsim 4 L^{*}$ local ellipticals had stopped forming stars by $z \gtrsim 1.2$, but that the progenitors of less massive ellipticals (down to $L^{*}$ ) can still show significant star formation at this epoch.
\end{abstract}

Key words: cosmology: observations - galaxies: elliptical and lenticular, cD - galaxies: evolution - galaxies: formation - galaxies: high-redshift - galaxies: starburst - galaxies: statistics - large-scale structure of universe

Online-only material: color figures

\section{INTRODUCTION}

The pioneering $K$-band near-infrared survey of Elston et al. (1988) was the first to identify galaxies with red colors of $(R-K)>5$ and hypothesized these to be either a new class of galaxy at $z>6$ or ellipticals at $z>1$. Graham \& Dey (1996) named these galaxies extremely red objects (EROs) for their extreme optical to infrared (IR) colors (e.g., $(R-K)_{\text {Vega }}>5.0$, $\left.(I-K)_{\text {Vega }}>4.0,(R-[3.6])_{\text {Vega }}>6.5\right)$ and recorded the first redshift for an ERO of $z=1.44$. We now know that the class of EROs consists of two $z>0.8$ sub-populations: galaxies with old red stellar populations (e.g., Spinrad et al. 1997) or star-forming galaxies with strong dust obscuration (e.g., from interaction and/or orientation; Dey et al. 1999; Yan \& Thompson 2003).

Since the highest mass galaxies at this redshift are often found to be EROs $\left(\gtrsim 10^{11.5} M_{\odot}\right.$, e.g., Moustakas et al. 2004; Conselice et al. 2008), and because EROs also exhibit very strong clustering, comparable to the local clustering of massive elliptical galaxies (e.g., Daddi et al. 2000, 2002; Brown et al. 2005), EROs have been posited as the progenitors of massive local ellipticals. This is important because the formation history of massive galaxies provides a crucial test of galaxy formation models. A key part of any such test is to link high- and low-redshift galaxy populations, and with the advent of large- area IR surveys, EROs provide an ideal and easily accessible testing ground for this purpose (Gonzalez-Perez et al. 2009).

One powerful means of linking galaxy populations across redshift is through clustering and the (dark matter) halo occupation model (e.g., Moustakas \& Somerville 2002). In the case of massive dark matter halos, simulations of dark matter structure evolution such as the Millennium Run (Springel et al. 2005) demonstrate that massive halos evolve rapidly in mass but little in (comoving) space over cosmic time. Since the most massive galaxies occupy the most massive halos (e.g., Seljak 2000), they too grow in mass but their (comoving) spatial distribution evolves slowly. This means that accurate measurements of ERO spatial clustering and host halo mass can be used to link these massive $z \gtrsim 0.8$ galaxies with local galaxy populations.

Previous studies have all found that EROs are very strongly clustered (e.g., Daddi et al. 2000, 2002; Firth et al. 2002; Roche et al. 2002; Brown et al. 2005; Georgakakis et al. 2005; Kong et al. 2006, 2009; Kim et al. 2011). However, the existing estimates of the clustering strengths, as measured by the scale $r_{0}$ where the two-point correlation strength is unity, show a broad range $\left(r_{0}=5.5-17 h^{-1} \mathrm{Mpc}\right)$ that makes it difficult to link the higher redshift EROs with local galaxy populations. Many of these discrepancies are likely due to differing selection criteria and cosmic variance due to small survey areas and sample 
sizes. Differences in methodology between studies, such as redshift distribution models and uncertainty estimates, are also possible contributors. We discuss the consequences of these factors below.

The diversity in ERO selection criteria is largely due to pragmatic choices for selecting high-redshift galaxy populations in different data sets. The present range of ERO selection criteria include (but are not limited to) $(R-K)_{\text {Vega }}>5.0$ (e.g., Brown et al. 2005), $(I-K)_{\text {Vega }}>4.0$ (e.g., Kong et al. 2009), $(R-H)_{\text {Vega }}>4.0$ (e.g., Firth et al. 2002), and $(R-[3.6])_{\text {Vega }}>$ 6.5 (e.g., Wilson et al. 2004). The consequences of using different ERO selection criteria have been considered in some studies (e.g., Yan \& Thompson 2003; Wilson et al. 2004; Conselice et al. 2008; Kim et al. 2011). Conselice et al. (2008) found that $\approx 60 \%$ of EROs were selected by both $(I-K)$ and $(R-K)$ criteria, with the main difference being a shift in the mean redshift $(\bar{z})$. Similarly, Kim et al. (2011) attribute the difference in clustering between $(r-K)$ and $(i-K)$ selected EROs to being a consequence of their difference in $\bar{z}$. Wilson et al. (2004) replaced the $K$ band with IRAC $3.6 \mu \mathrm{m}$ and suggested that ERO samples selected by this color will include more dusty EROs with star formation than $K$-band-selected EROs (as we will discuss in Section 3.2).

Morphological and spectroscopic studies of EROs show them to be a mixture of galaxies with and without star formation (e.g., Smail et al. 2002; Moustakas et al. 2004; Stern et al. 2006; Wilson et al. 2007; Conselice et al. 2008; Kong et al. 2009). The relative fractions of dusty galaxies with active star formation (hereafter, star-forming) and galaxies dominated by old stellar populations and little ongoing star formation (hereafter, passive) depend on the ERO selection criteria and the limiting magnitude (e.g., Moustakas et al. 2004; Conselice et al. 2008). The sub-populations can be very broad, as star-forming EROs can have star formation rates (SFRs) of between 10 and $200 M_{\odot} \mathrm{yr}^{-1}$ (e.g., Messias et al. 2010), but distinguishing further between sub-populations of star-forming EROs is beyond the scope of this study. Separating passive from star-forming EROs is important because we should not expect the two ERO types to possess the same stellar mass, occupy the same mass dark matter halos, or be the progenitors of the same local galaxy populations.

Different fractions of these two ERO sub-populations selected by different ERO criteria may well explain the large differences seen in ERO clustering studies. Unfortunately, accurate morphological and spectroscopic classifications are often unavailable for the sources used in ERO clustering studies. In most cases, colors are used to distinguish between star-forming and passive EROs. NIR colors (e.g., $(J-K)$; Pozzetti \& Mannucci 2000; Kim et al. 2011) cannot adequately distinguish between the ERO types if they do not bracket or straddle the $4000 \AA$ break, which does not work for all EROs (only $z \gtrsim 1.4$ ) and requires high-precision photometry (e.g., Stern et al. 2006). The mid-IR color techniques introduced by Stern et al. (2006) ([3.6] - [24]) and Wilson et al. (2007) ([3.6] - [8.0]) are more promising because they use the emission from hot dust in star-forming galaxies to obtain a better separation of the two ERO types. However, these mid-IR color techniques require space observatory imaging, which is not always available.

Accurate spatial clustering measurements require reliable redshift distributions, but there are relatively few EROs with spectroscopic redshifts (e.g., Graham \& Dey 1996; Dey et al. 1999; Cimatti et al. 2002; Conselice et al. 2008), and spectroscopic samples at high redshift are vastly incomplete (e.g., Cooper et al. 2007). Typically, redshift distributions are assumed to have a particular shape, are derived from photometric red- shifts, or both. This can be a problem because spatial clustering analyses are strongly dependent on the shape of the redshift distribution, contributing to the spread in previous clustering estimates. Unfortunately, using photometric redshifts and models of the redshift distribution remain the best methodology available at present.

An additional problem is that the angular correlation function $(w(\theta))$ is usually fit as a fixed power law, which may not correctly represent the data even if it is the only option given the sample sizes (e.g., Firth et al. 2002; Georgakakis et al. 2005). Furthermore, the uncertainties in the correlation function are frequently assumed to be Poisson with no correlations between data points (e.g., Kong et al. 2009; Kim et al. 2011). An incorrect power law, possibly combined with a poor uncertainty model, can easily result in incorrect estimates of the spatial correlation length. A likely consequence of these problems is the broad range of ERO clustering estimates that are consistent with their having been the progenitors of anything from local $L^{*}$ ellipticals to Abell-type clusters (see Norberg et al. 2002; Abadi et al. 1998 for corresponding local galaxy population $r_{0}$ values, respectively).

In this paper, we present a comprehensive measurement of ERO clustering. The sample sizes and survey area are large enough to address all these problems. We consider the largest ERO samples to date, selected in either the $K_{s}$ or IRAC [3.6] bands over $7.80 \mathrm{deg}^{2}$ or $8.53 \mathrm{deg}^{2}$ of the NOAO Deep Wide Field Survey (NDWFS) Boötes field (Jannuzi \& Dey 1999), respectively. The NDWFS Boötes field provides a factor of three larger survey area than any previous ERO clustering study. We study three ERO samples using the selection criteria $\left(R-K_{s}\right)>$ 5.0, $\left(I-K_{S}\right)>4.0$, and $(I-[3.6])>5.0$, in an attempt to establish the differences and similarities between these samples and to better understand previous results. Our ERO samples contain 28,724 $\left(R-K_{S}\right)>5.0,22,451\left(I-K_{S}\right)>4.0$, and $64,370(I-[3.6])>5.0$ selected EROs, down to magnitude limits of $K_{s}<19.4, K_{s}<19.4$, and [3.6] $<18.5$, respectively. The sample sizes are 5 times larger than any previous study and 10 times larger than our previous study of EROs in Boötes (Brown et al. 2005).

We use optical NDWFS $B_{w} R I$ photometry (Jannuzi \& Dey 1999), spectra from the active galactic nuclei (AGNs) and Galaxy Evolution Survey (AGES; Kochanek et al. 2012), and various small projects with instruments including Keck, Gemini, and the Kitt Peak National Observatory Mayall $4 \mathrm{~m}$, in conjunction with IRAC photometry from the Spitzer Deep Wide-Field Survey (SDWFS; Ashby et al. 2009), NEWFIRM $K_{s}$-band photometry (A. Gonzalez et al., in preparation), and MIPS $24 \mu \mathrm{m}$ photometry from the MIPS AGN and Galaxy Evolution Survey (MAGES; Jannuzi et al. 2010). We test mid-infrared ERO separation techniques utilizing equivalent $B_{w} R I K_{s}$ and IRAC photometry (Bundy et al. 2006; Dey et al. 2007; Barmby et al. 2008), MIPS photometry from the FarInfrared Deep Extragalactic survey (FIDEL; Dickinson \& FIDEL team 2007), and DEEP2 spectroscopic indicators and one-dimensional spectra (Yan et al. 2009; Newman et al. 2012, and references therein) in the Extended Groth Strip (EGS).

This paper is structured as follows. We describe the data sets, photometry, and photometric redshift estimation in Section 2. Our ERO samples are defined and our ERO separation technique is described in Section 3. In Section 4 we present our clustering analysis and results, followed by a discussion and summary in Section 5. Throughout this paper, unless otherwise specified, all magnitudes are in the Vega magnitude system. Spitzer IRAC and 
MIPS magnitudes are quoted using square brackets (e.g., $3.6 \mu \mathrm{m}$ is [3.6]). We introduce the following nomenclature for our ERO samples: $\left(R-K_{S}\right)>5.0,\left(I-K_{S}\right)>4.0$, and $(I-[3.6])>5.0$ selected ERO samples are respectively referred to as the $\mathrm{ERO}_{R K}$, $\mathrm{ERO}_{I K}$, and $\mathrm{ERO}_{I 3.6}$ samples. We assume a standard $\Lambda \mathrm{CDM}$ flat cosmology, with $\Omega_{m}=0.24, \Omega_{\Lambda}=0.76, \sigma_{8}=0.76, h=0.73$, and $H_{0}=100 \mathrm{hm} \mathrm{s}^{-1} \mathrm{Mpc}^{-1}$ (Spergel et al. 2007).

\section{DATA}

\subsection{Object Catalogs}

Our galaxies were selected from optical $\left(B_{w} R I\right)$ and IR imaging $(3.6-24.0 \mu \mathrm{m})$ in $8.53 \mathrm{deg}^{2}$ of the Boötes field by the NDWFS, SDWFS, and MAGES surveys. Where available, we add the NEWFIRM $K_{s}$-band imaging of $7.80 \mathrm{deg}^{2}$ of the Boötes field. Any samples selected using the $K_{s}$ band are limited to this reduced field size. The wide-area AGES survey (Kochanek et al. 2012) provides redshifts for the brightest EROs in the field, while the spectra for the fainter EROs come from various small projects using Keck, Gemini, and the Kitt Peak National Observatory Mayall $4 \mathrm{~m}$ telescope.

Source catalogs were generated by running SExtractor (Bertin $\&$ Arnouts 1996) in single-image mode on both the $I$ band and $4.5 \mu \mathrm{m}$ images. Catalogs were merged after accounting for astrometric offsets. We make a pragmatic choice to use the $4.5 \mu \mathrm{m}$ selected catalog for source positions and photometry, as this catalog was readily available and better represents $3.6 \mu \mathrm{m}$ sources than $I$ band. This is because objects can be so red as to be detected at $4.5 \mu \mathrm{m}$ but not in the optical. The colors of $[3.6]<18.5$ galaxies span $0 \lesssim[3.6]-[4.5] \lesssim 1$, so sources with [3.6] $=18.5$ will have a magnitude of $17.5 \lesssim[4.5] \lesssim 18.5$ and will be comfortably detected in the SDWFS $4.5 \mu \mathrm{m}$ selected catalog since it is $80 \%$ complete at [4.5] $=18.1$ and has a $5 \sigma$ depth of [4.5] $\approx 18.5$.

Photometry in all other bands is measured at the $4.5 \mu \mathrm{m}$ detected source positions. For the case of objects detected in the $4.5 \mu \mathrm{m}$ selected catalog but with non-detections in $I$ band and $R$ band, we assign conservative upper limits of $I=24$ and $R=25$. These magnitude limits are sufficiently bright so that there are no variations in the galaxy distribution due to the varying depths of the different pointings. We have tested this by inspecting the uniformity of the galaxy distribution for faint magnitude slices about these assigned limits. Our three ERO criteria are shown as applied to the $4.5 \mu \mathrm{m}$ source catalog (with imaging quality masks applied to remove unusable areas of the Boötes field) in Figure 1.

We measured aperture photometry using our own analysis code, which estimates uncertainties using a Monte Carlo approach. The optical and near-IR images were smoothed to a common point-spread function (PSF) of 1".35 (corresponding to the worst seeing) in the $B_{w} R I H K$ bands and 1".60 in $J$ band, prior to running the photometry code, so that the fraction of the flux measured with small aperture photometry did not vary with position. The fluxes were PSF-corrected and measured using multiple aperture diameters of $4^{\prime \prime}-10^{\prime \prime}$ in $1^{\prime \prime}$ steps, corresponding to physical distances of $\approx 24-160 h^{-1} \mathrm{kpc}$ at $z=1.0$, respectively. We then assign the default aperture size for color and magnitude measurements using a sliding scale based on the $6^{\prime \prime}$ aperture $I$-band apparent magnitude, where fluxes for objects with $I>23.0$ are measured through a $4^{\prime \prime}$ aperture and the fluxes for objects with $I<20$ are measured through $10^{\prime \prime}$ apertures, which are sufficient to mitigate aperture bias. Astrometric offsets between bands were measured and corrected. The residuals

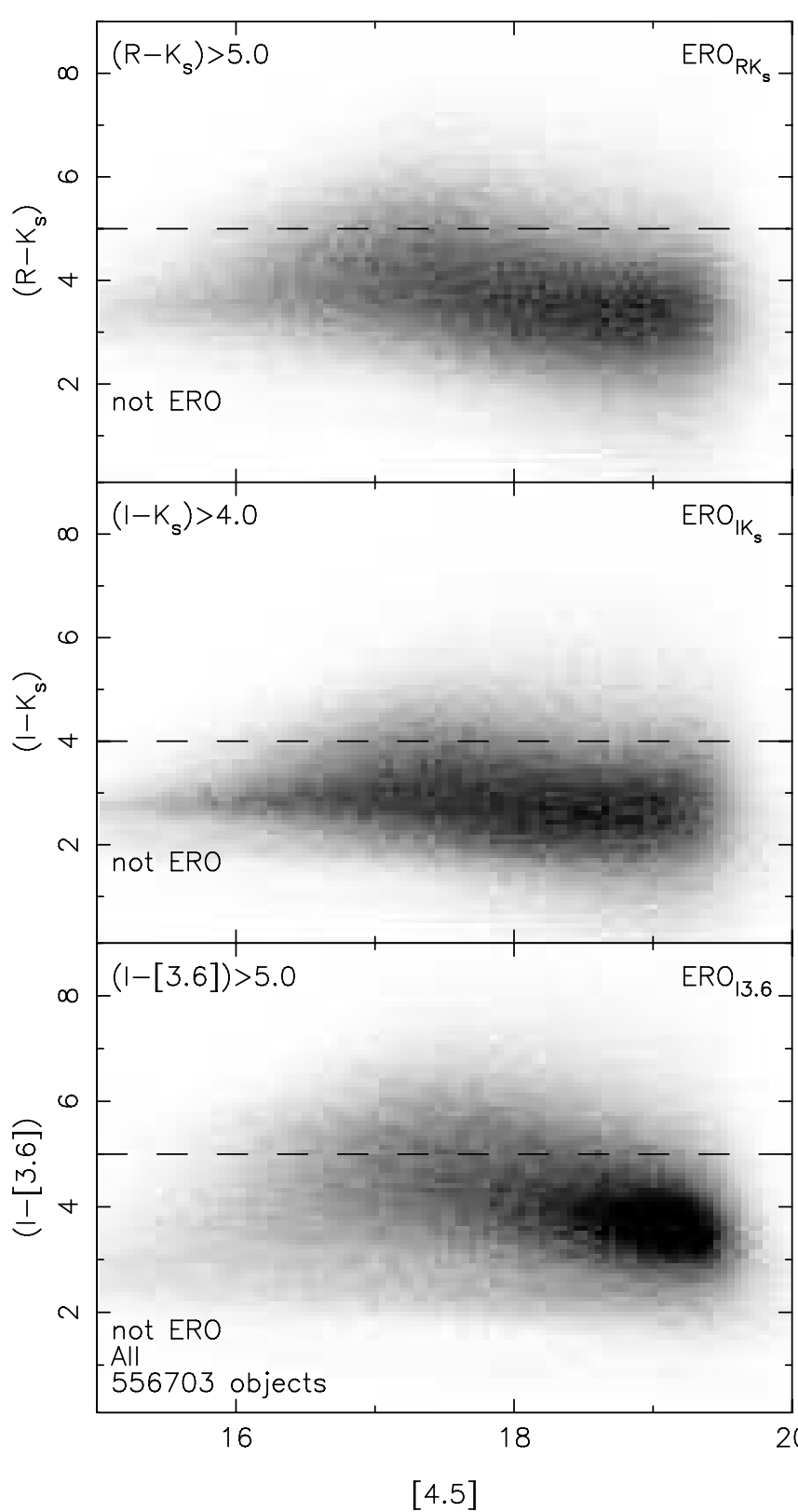

Figure 1. ERO sample selection from the uncleaned $4.5 \mu \mathrm{m}$ selected catalog. Top to bottom, $\left(R-K_{s}\right),\left(I-K_{s}\right)$, and $(I-[3.6])$ color for the entire $4.5 \mu \mathrm{m}$ selected catalog in the NDWFS Boötes field. Our ERO selection criteria $\left(R-K_{s}\right)>5.0,\left(I-K_{s}\right)>4.0$, and $(I-[3.6])>5.0$ are shown by the dashed lines.

were far smaller than our aperture size, making them negligible. Jannuzi \& Dey (1999) and Brown et al. (2007) provide additional details for the NDWFS data, and Ashby et al. (2009) provide details for the SDWFS IRAC photometry.

The $24 \mu \mathrm{m}$ fluxes were measured using PSF photometry on the MAGES images (Jannuzi et al. 2010) for each source in the $4.5 \mu \mathrm{m}$ selected catalog, with uncertainties determined using a Monte Carlo method. The NEWFIRM $K_{s}$ catalogs are described in A. Gonzalez et al. (in preparation). We used an exposure time-weighted map to exclude poor photometry in the $K_{s}$ band, and we only use photometry from regions where the exposure map was greater than $40 \%$ of the maximum for the NEWFIRM images. Aperture diameters for the NEWFIRM $K_{s}$ data were determined as described above. 


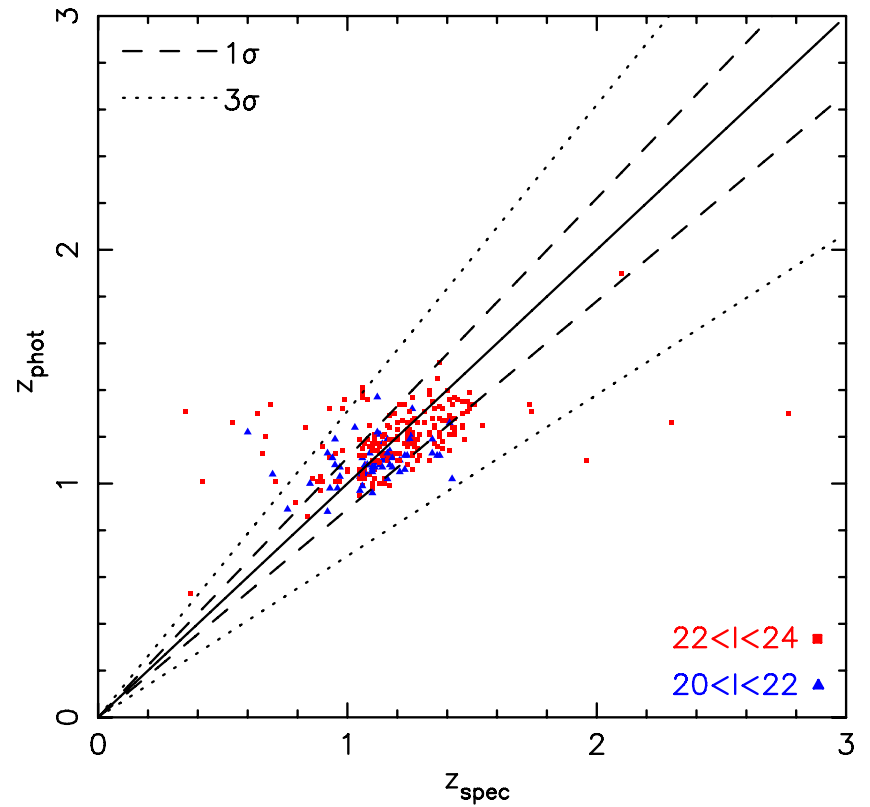

Figure 2. $z_{\text {phot }}$ vs. $z_{\text {spec }}$ for $273 \mathrm{ERO}_{I 3.6}$ galaxies in the NDWFS Boötes field with both photometric $\left(z_{\text {phot }}\right)$ and spectroscopic redshift $\left(z_{\mathrm{spec}}\right)$ estimates. Sixty-eight percent of our $z_{\text {phot }}$ estimates for $20<I<24$ EROs are within $11 \%$ (indicated by the dashed lines) of their corresponding spectroscopic redshift $\left(z_{\text {spec }}\right)$, or $\sigma_{z} /(1+z) \approx 0.06$. The results are similar for $20<I<22$ (triangles) and $22<I<24$ (squares), which demonstrate reasonable stability in $z_{\text {phot }}$ accuracy between $20<I<24$. The fraction of catastrophic outliers, defined as when $\Delta_{z}=\left|z_{\text {phot }}-z_{\text {spec }}\right|>3 \sigma_{z}$, is $\approx 6 \%$.

(A color version of this figure is available in the online journal.)

To test some of the techniques used in this paper, we also employ 6285 galaxies observed through identical $B_{w} R I$ (Dey et al. 2007), $K_{s}$ (Bundy et al. 2006), IRAC (Barmby et al. 2008), MIPS (FIDEL; Dickinson \& FIDEL team 2007) filters, and with DEEP2 DR3 spectra (Yan et al. 2009; Newman et al. 2012, and references therein) from the EGS. Photometry for these catalogs was measured and matched as described above.

\subsection{Photometric Redshifts}

Photometric redshifts $\left(z_{\text {phot }}\right)$ were estimated using the artificial neural network based $\mathrm{ANN}_{z}$ code (Firth et al. 2003). Our spectroscopic training set was largely derived from AGES (Kochanek et al. 2012), various small NDWFS projects, and DEEP2 spectroscopy of the EGS (Yan et al. 2009). At $z>0.6$ and $I>20.5$, there are relatively few galaxies, so we expanded the training set by making fainter copies of bright galaxies with modified colors (to preserve color-magnitude relations). Objects with colors outside the range of the $z<1.6$ training set were not assigned a $z_{\text {phot }}$, as $\mathrm{ANN} z$ has a limited ability to extrapolate. Objects with $z_{\text {spec }}>1.6$ that are assigned a $z_{\text {phot }}$ have systematically underestimated $z_{\text {phot }}$ for the same reason, as can be seen in Figure 2.

Figure 2 demonstrates the accuracy of our $z_{\text {phot }}$ estimation for $273 \mathrm{ERO}_{\text {I3.6 }}$ galaxies. These galaxies were included in the $\mathrm{ANN} z$ training set, which allowed it to span the color of EROs. We find that $68 \%$ of our $z_{\text {phot }}$ estimates for $20<I<24$ EROs are within $11 \%$ of their corresponding spectroscopic redshift $\left(z_{\text {spec }}\right)$, or $\sigma_{z} /(1+z) \approx 0.06$. The $z_{\text {phot }}$ were determined using our $I$-band-selected catalog out to $I<23.5$ and later matched to objects in the $4.5 \mu \mathrm{m}$ selected catalog by astrometry. Due to the limited number of $z_{\text {spec }}$ available for our EROs, we cross-checked our $z_{\text {phot }}$ against the template $z_{\text {phot }}$ of Brodwin et al. (2006) and find good agreement between the two catalogs.
The fraction of catastrophic $z_{\text {phot }}$ errors, which we define as when $\Delta_{z}=\left|z_{\text {phot }}-z_{\text {spec }}\right|>3 \sigma_{z}$, is $\approx 6 \%$. At $z \approx 0.5$, stellar spectral features around $\approx 2.3 \mu \mathrm{m}$ produce red ([3.6] - [4.5]) colors. This can confuse $\mathrm{ANN} z$ and is likely responsible for the systematic overestimation of $z_{\text {phot }}$ for objects with $0.4<$ $z_{\text {spec }}<0.7$ shown in Figure 2 . The likely reason for systematic $z_{\text {phot }}$ underestimates of objects with $z_{\mathrm{spec}}>1.6$ is as described earlier.

We note that our spectra do not uniformly cover the magnitude and redshift range of our sample. There are very few $z>1.5$ galaxy spectra in Boötes and a large number of $z<0.8$ spectra from AGES. Since the very red nature of EROs limits the redshift of our sample to $z \gtrsim 0.8$, the fraction of $z \approx 0.5$ galaxies contaminating our sample is probably overestimated in Figure 2. We discuss our method for managing these $z_{\text {phot }}$ errors, as well as our method for estimating the overall redshift distributions for our samples, in Section 4.2.

\subsection{Star and Quasar Removal}

AGES spectra were used to identify 241 stars and 464 quasars (QSOs) in our $4.5 \mu \mathrm{m}$ selected catalog. Some of the AGES selected stars will have peculiar colors because they were targeted as candidate AGNs (e.g., X-ray sources). We used the $(R-I)$ and $(I-[3.6])$ color distribution of these stars to define a cut to remove these from our ERO samples. This star removal cut, of sources with $(R-I)>0.46(I-[3.6])-$ 0.26 and with $(I-[3.6])<5.1$, which is shown in Figure 3, removes 58,790 objects from the $4.5 \mu \mathrm{m}$ selected catalog. Figure 3 demonstrates that due to the extremely red color of EROs, this star cut excludes $100 \%$ of stars with a negligible impact on the ERO samples.

There are multiple IRAC color AGN selection criteria in the literature (e.g., Lacy et al. 2004; Stern et al. 2005). Due to the ([3.6] - [4.5]) colors of $z \gtrsim 1$ galaxies, the Stern et al. (2006) AGN criterion deliberately removes a large number of EROs. Using the colors of AGES identified quasars, we demonstrate in Figure 4 the effectiveness of the IRAC color selection introduced by Lacy et al. (2004) for the ERO samples. Figure 4 shows that quasars selected by our ERO criteria occupy a region of color space corresponding to a distinct tail within the Lacy et al. (2004) AGN region. We have modified the Lacy et al. (2004) color criteria to conservatively select AGNs in this distinct tail seen above the ERO populations in Figure 4. It is clear that these selection criteria are not $100 \%$ effective, but our aim is to select a complete ERO population, not a complete sample of AGNs. The AGN cut removes 71,664 AGNs from the $4.5 \mu \mathrm{m}$ selected catalog.

After application of both of these stellar and QSO removal cuts, our $4.5 \mu \mathrm{m}$ source catalog is reduced from 556,703 to 426,249 objects. Since the $K_{s}$-band photometry was filtered by an exposure time weight map, the $4.5 \mu \mathrm{m}$ source catalog including good $K_{s}$-band photometry is reduced to 366,070 objects.

\section{SAMPLES}

\subsection{ERO Selection}

From our cleaned $4.5 \mu \mathrm{m}$ source catalogs, the $\mathrm{ERO}_{R K}$, $\mathrm{ERO}_{I K}$, and $\mathrm{ERO}_{I 3.6}$ cuts respectively select $16 \%, 17 \%$, and $27 \%$ of all $4.5 \mu \mathrm{m}$ detected sources. From 60,066, 63,798, and $113,0984.5 \mu \mathrm{m}$ selected $\mathrm{ERO}_{R K}, \mathrm{ERO}_{I K}$, and $\mathrm{ERO}_{I 3.6}$ galaxies, we selected 28,724, 22,451, and 64,370 EROs with $K_{s}<19.4$, $K_{s}<19.4$, and [3.6] $<18.5$, respectively. Sample magnitude 


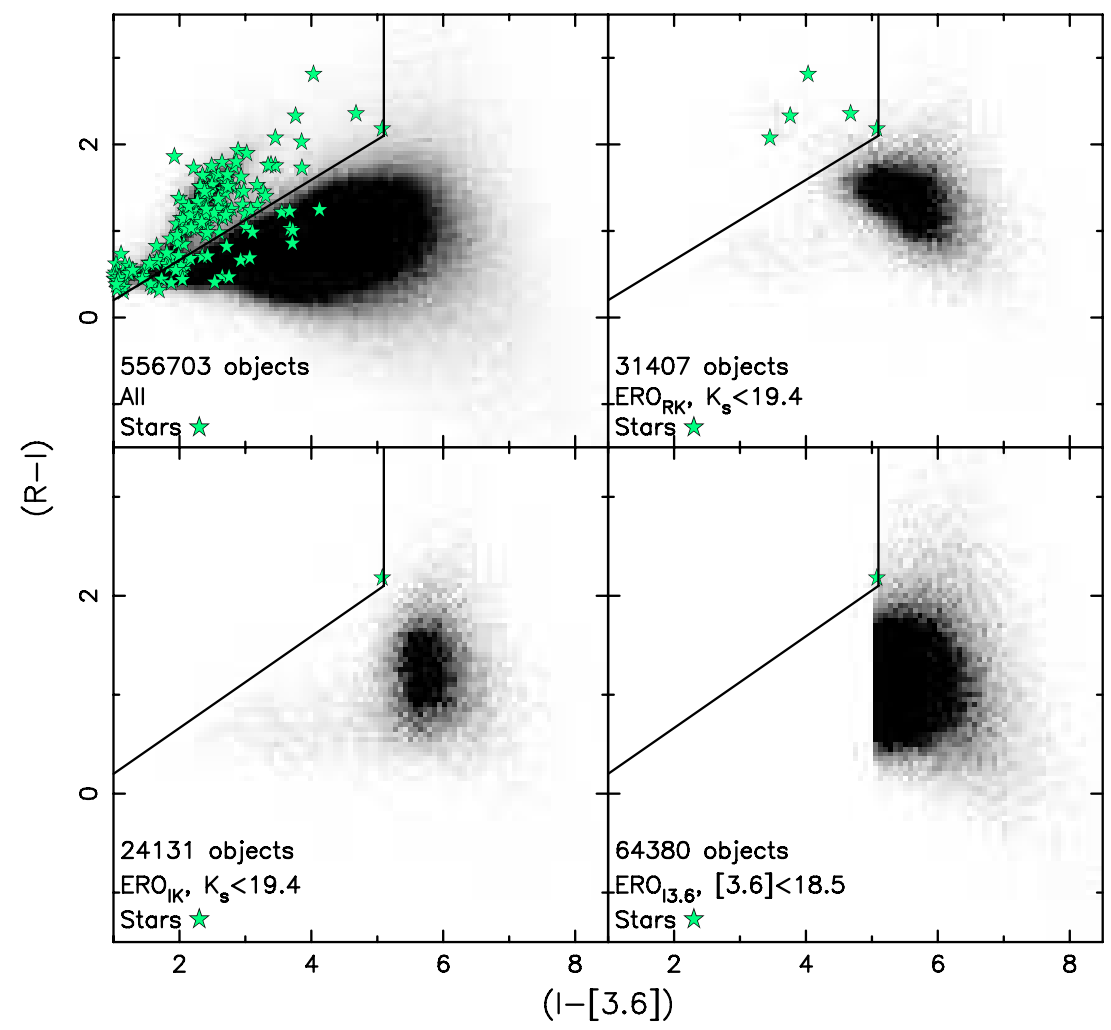

Figure 3. Control of stellar contamination shown for the uncleaned $4.5 \mu \mathrm{m}$ selected catalog. $(R-I)$ vs. $(I-[3.6])$ for $\mathrm{All}, \mathrm{ERO}_{R K}$, $\mathrm{ERO}_{I K}$, and $\mathrm{ERO}_{I 3.6}$ objects in the NDWFS Boötes field. Green stars are AGES spectroscopically identified stars. We use the location of these stars to place an empirical cut to remove stars from our ERO samples. The stellar cut combined with the intrinsically red ( $I-[3.6])$ color of EROs keeps the samples free from stellar contamination.

(A color version of this figure is available in the online journal.)

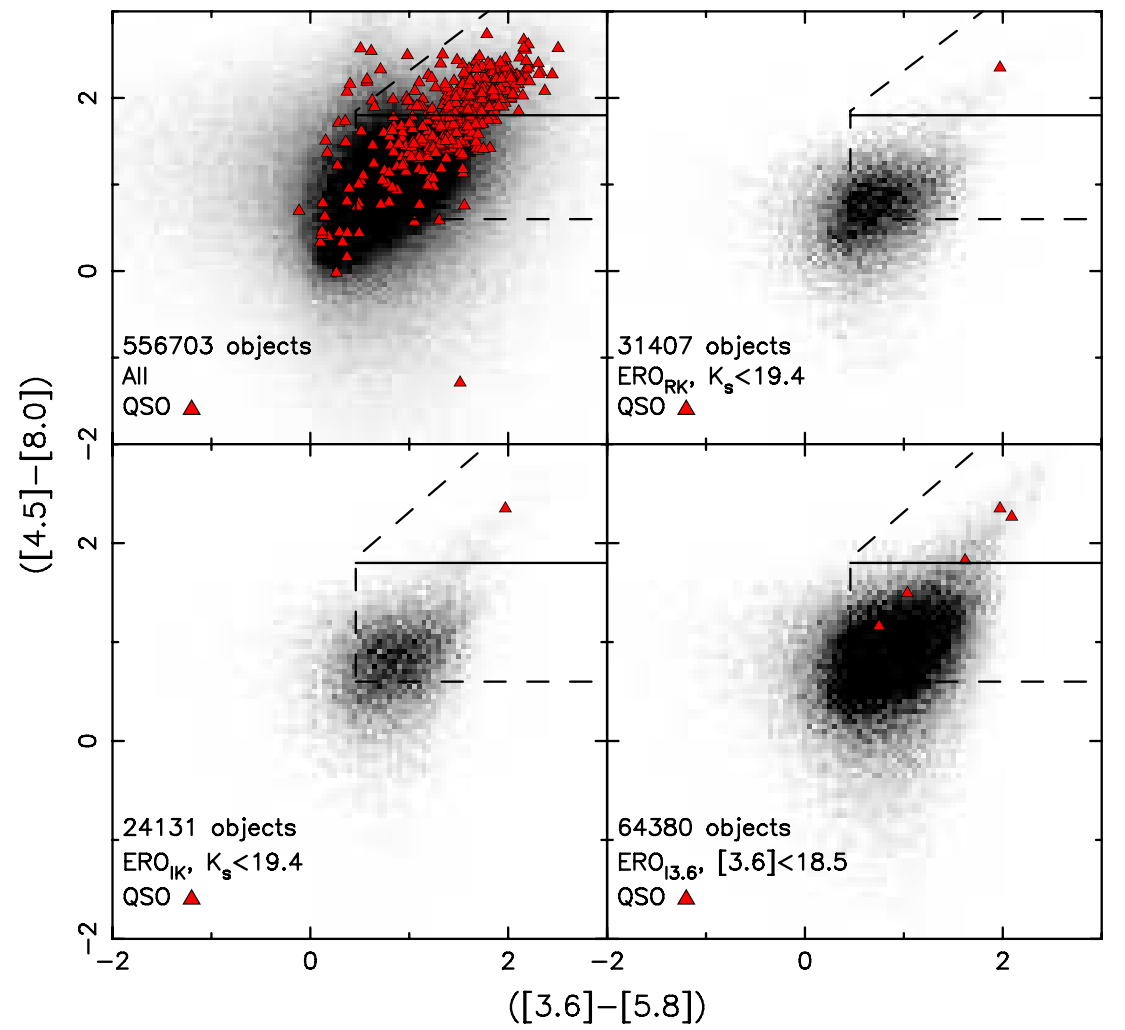

Figure 4. Control of quasar contamination shown for the uncleaned $4.5 \mu \mathrm{m}$ selected catalog. A modified form of the Lacy et al. (2004) mid-IR, ([4.5] - [8.0]) vs. ([3.6] - [5.8]) AGN selection region is used to minimize quasar contamination within our sample. This is shown for $\mathrm{All}, \mathrm{ERO}_{R K}, \mathrm{ERO}_{I K}$, and $\mathrm{ERO}_{I 3.6}$ objects in the NDWFS Boötes field. AGES spectroscopically identified QSOs are shown with red triangles, and we use these to place a more conservative empirical cut at $([4.5]-[8.0]) \geqslant 1.8$ (solid line) to remove obvious AGN contaminants from our sample. The original Lacy et al. (2004) criteria are shown by the dashed lines.

(A color version of this figure is available in the online journal.) 


\section{[3.6]}

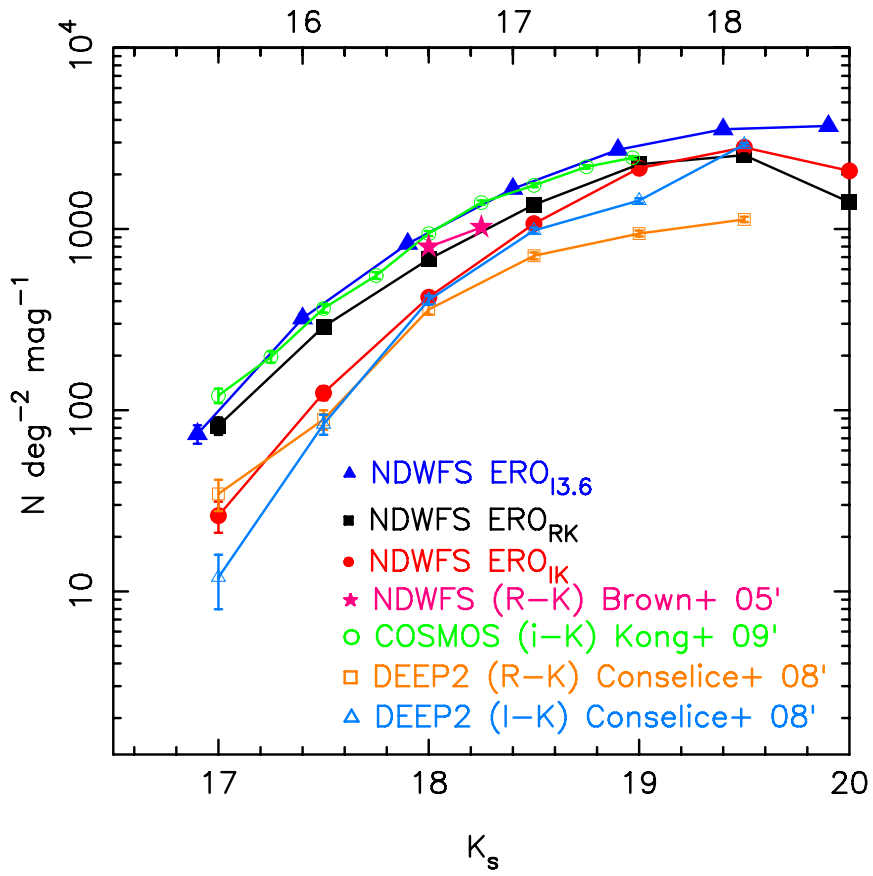

Figure 5. Completeness-corrected surface densities $(N)$ for each ERO sample. For comparison with the surface densities found in previous ERO studies we show the distributions from Kong et al. (2009) $((i-K)>3.96)$, Brown et al. (2005) $((R-K)>5.0)$, and Conselice et al. (2008) $((R-K)>5.3$ and $(I-K)>4.0)$. The error bars are included for all points but in many cases are too small to be seen. We use $K_{s} \approx[3.6]+1.4$ to shift [3.6] magnitudes onto the $K_{s}$ scale, based on the average of $K_{s}-$ [3.6] for our EROs.

(A color version of this figure is available in the online journal.)

limits were set to correspond to the flattening in surface density $(N)$ seen in Figure 5. SDWFS is $80 \%$ complete at [3.6] $=18.2$, and this limit is also consistent with our $5 \sigma I$ band and [3.6] detection limits (Jannuzi \& Dey 1999; Ashby et al. 2009). Throughout this paper we take $K_{s} \approx[3.6]+1.4$, based on the average of $K_{s}-$ [3.6] for our EROs.
The fraction of $4.5 \mu \mathrm{m}$ selected sources meeting the $\mathrm{ERO}_{I 3.6}$ criterion was found to increase with increasing limiting magnitude, from $19 \%$ at [3.6] $<17.5$ to $57 \%$ at [3.6] $<18.5$. Similarly, the respective fractions of $4.5 \mu \mathrm{m}$ selected sources meeting the $\mathrm{ERO}_{R K}$ and $\mathrm{ERO}_{I K}$ criteria were also found to increase with increasing limiting magnitude from $11 \%$ and $6 \%$ at $K_{s}<18.4$ to $48 \%$ and $35 \%$ at $K_{s}<19.4$. The fractional increase in EROs with increasing apparent magnitude for the $\mathrm{ERO}_{R K}$ and $\mathrm{ERO}_{I K}$ samples is $29 \%-37 \%$. The $\mathrm{ERO}_{I 3.6}$ sample has a fractional increase of $38 \%$ and selects a much larger number of high-redshift galaxies than both $\mathrm{ERO}_{R K}$ and $\mathrm{ERO}_{I K}$ samples. The $\mathrm{ERO}_{R K}$ criterion selects a greater fraction than the $\mathrm{ERO}_{I K}$ criterion, which is likely due to the difference in mean redshift between these two samples.

As shown in Figure 5, the surface density of the $\mathrm{ERO}_{R K}$ sample is consistent with that of our previous study of a subfield of Boötes for the same selection criteria (Brown et al. 2005). Furthermore, if we raise our criteria to $\left(R-K_{s}\right)>5.3$ to match that of Conselice et al. (2008), we find good agreement in surface density. Likewise, we see good agreement between our $\mathrm{ERO}_{I K}$ sample and the $(I-K)>4.0$ EROs of Conselice et al. (2008). To compare with the $(i-K)>3.96$ EROs of Kong et al. (2009), we tested the surface density of $\left(I-K_{s}\right)>3.7$ (which roughly corresponds to $(i-K)>3.96$ ) objects and also found good agreement in surface densities.

In Figure 6, we show the model redshift distributions for EROs out to the magnitude limits for each of our samples. The mean redshift $(\bar{z})$ of the $\mathrm{ERO}_{R K}$ sample, $\bar{z}=1.13$, is lower than that of the $\mathrm{ERO}_{I K}$ sample, with $\bar{z}=1.19$, in agreement with previous work (e.g., Conselice et al. 2008; Kim et al. 2011). We find that the $\mathrm{ERO}_{I 3.6}$ sample has a very similar redshift distribution to the $\mathrm{ERO}_{I K}$ sample, with $\bar{z}=1.17$, although with far greater number density. Since all our samples contain at least $\gtrsim 9000$ objects with photometric redshifts, the statistical uncertainties in the mean redshift are negligible $(<1 \%)$, and we are dominated by systematic uncertainties.

Figures 3 and 4 illustrate in color space the similarities and differences between the $\mathrm{ERO}_{I 3.6}, \mathrm{ERO}_{R K}$, and $\mathrm{ERO}_{I K}$ samples. We see that there is significant overlap between the samples

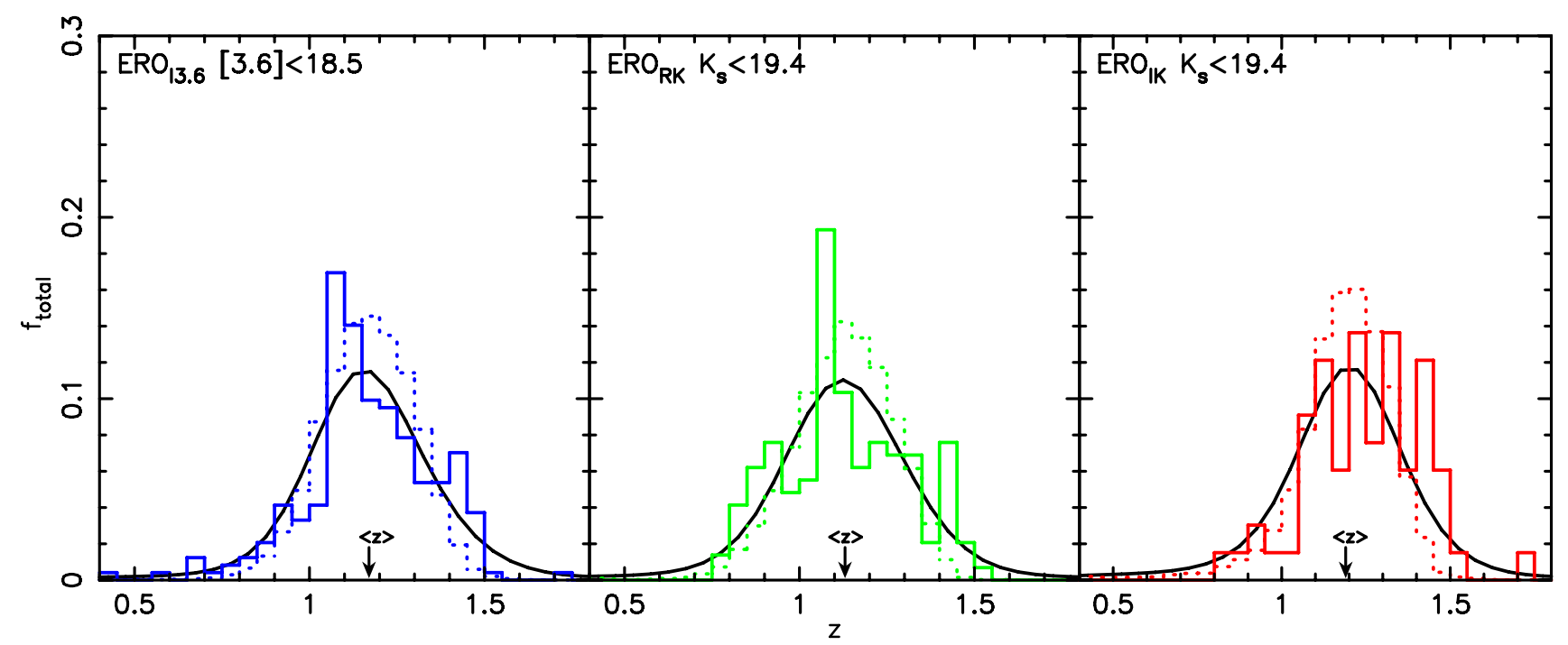

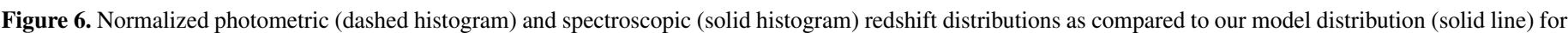

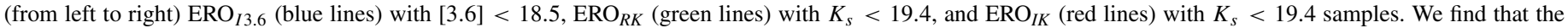

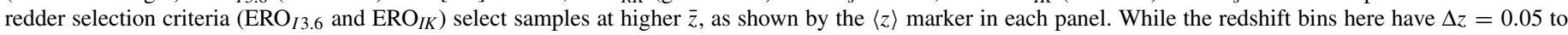
reduce the noise for the spectroscopic redshifts, $\Delta z=0.02$ is used in our analysis.

(A color version of this figure is available in the online journal.) 
Table 1

Summary of Combined ERO Clustering Results

\begin{tabular}{|c|c|c|c|c|c|c|c|c|c|}
\hline ERO Cut & Mag. Limit & ERO Type & $N$ & $\bar{z}$ & $\gamma$ & $w\left(1^{\prime}\right)$ & $\chi^{2} /$ dof & $\begin{array}{c}r_{0} \\
\left(h^{-1} \mathrm{Mpc}\right)\end{array}$ & $\begin{array}{c}M_{\text {halo }} \\
\left(\times 10^{12} M_{\odot}\right)\end{array}$ \\
\hline$I-[3.6]>5.0$ & {$[3.6]<16.5$} & ALL & 3209 & 1.11 & $1.91 \pm 0.08$ & $0.295 \pm 0.028$ & 8.48 & $8.77 \pm 1.65$ & $6.7_{-2.9}^{+4.8}$ \\
\hline$I-[3.6]>5.0$ & {$[3.6]<17.0$} & ALL & 9537 & 1.14 & $1.98 \pm 0.06$ & $0.182 \pm 0.013$ & 2.29 & $6.78 \pm 0.95$ & $2.9_{-1.0}^{+1.5}$ \\
\hline$I-[3.6]>5.0$ & {$[3.6]<17.5$} & ALL & 21800 & 1.16 & $1.89 \pm 0.05$ & $0.126 \pm 0.008$ & 2.93 & $6.10 \pm 0.77$ & $2.1_{-0.7}^{+1.0}$ \\
\hline$I-[3.6]>5.0$ & {$[3.6]<18.0$} & ALL & 40969 & 1.17 & $1.87 \pm 0.05$ & $0.085 \pm 0.005$ & 9.02 & $5.08 \pm 0.54$ & $1.1_{-0.4}^{+0.4}$ \\
\hline$I-[3.6]>5.0$ & {$[3.6]<18.5$} & ALL & 64370 & 1.17 & $1.76 \pm 0.02$ & $0.071 \pm 0.002$ & 22.92 & $4.93 \pm 0.21$ & $1.0_{-0.0}^{+0.2}$ \\
\hline$R-K_{s}>5.0$ & $K_{s}<17.9$ & ALL & 2715 & 1.05 & $1.77 \pm 0.07$ & $0.312 \pm 0.031$ & 2.95 & $11.21 \pm 2.08$ & $14.3_{-6.1}^{+9.9}$ \\
\hline$R-K_{s}>5.0$ & $K_{s}<18.4$ & ALL & 6801 & 1.09 & $1.80 \pm 0.06$ & $0.238 \pm 0.020$ & 3.86 & $9.63 \pm 1.57$ & $8.9_{-3.5}^{+5.3}$ \\
\hline$R-K_{s}>5.0$ & $K_{s}<18.9$ & ALL & 15013 & 1.12 & $1.75 \pm 0.06$ & $0.169 \pm 0.014$ & 3.46 & $8.43 \pm 1.39$ & $5.9_{-2.2}^{+3.6}$ \\
\hline$R-K_{s}>5.0$ & $K_{s}<19.4$ & ALL & 28724 & 1.13 & $1.83 \pm 0.06$ & $0.112 \pm 0.008$ & 7.34 & $6.26 \pm 0.93$ & $2.3_{-0.9}^{+1.3}$ \\
\hline$I-K_{s}>4.0$ & $K_{s}<17.9$ & ALL & 1314 & 1.08 & $1.58 \pm 0.10$ & $0.235 \pm 0.041$ & 2.80 & $12.68 \pm 3.14$ & $21.0_{-12.3}^{+20.0}$ \\
\hline$I-K_{s}>4.0$ & $K_{s}<18.4$ & ALL & 3672 & 1.15 & $1.78 \pm 0.08$ & $0.295 \pm 0.029$ & 3.85 & $11.19 \pm 2.27$ & $14.2_{-6.6}^{+11.0}$ \\
\hline$I-K_{s}>4.0$ & $K_{s}<18.9$ & ALL & 9829 & 1.18 & $1.71 \pm 0.07$ & $0.223 \pm 0.022$ & 6.92 & $9.98 \pm 2.00$ & $10.0_{-4.4}^{+7.6}$ \\
\hline$I-K_{s}>4.0$ & $K_{s}<19.4$ & ALL & 22451 & 1.19 & $1.75 \pm 0.07$ & $0.148 \pm 0.014$ & 9.83 & $7.62 \pm 1.35$ & $4.2_{-2.0}^{+2.6}$ \\
\hline$I-[3.6]>5.0$ & {$[3.6]<16.5$} & ALL & 3209 & 1.11 & 1.80 & $0.319 \pm 0.024$ & 8.21 & $10.14 \pm 0.76$ & $10.5_{-2.2}^{+2.6}$ \\
\hline$I-[3.6]>5.0$ & {$[3.6]<17.0$} & ALL & 9537 & 1.14 & 1.80 & $0.213 \pm 0.012$ & 3.09 & $8.53 \pm 0.48$ & $6.1_{-1.0}^{+1.1}$ \\
\hline$I-[3.6]>5.0$ & {$[3.6]<17.5$} & ALL & 21800 & 1.16 & 1.80 & $0.137 \pm 0.007$ & 2.73 & $6.81 \pm 0.35$ & $3.0_{-0.4}^{+0.5}$ \\
\hline$I-[3.6]>5.0$ & {$[3.6]<18.0$} & ALL & 40969 & 1.17 & 1.80 & $0.092 \pm 0.004$ & 7.69 & $5.53 \pm 0.24$ & $1.5_{-0.2}^{+0.2}$ \\
\hline$I-[3.6]>5.0$ & {$[3.6]<18.5$} & ALL & 64370 & 1.17 & 1.80 & $0.073 \pm 0.003$ & 13.90 & $4.90 \pm 0.20$ & $1.0_{-0.1}^{+0.1}$ \\
\hline$R-K_{s}>5.0$ & $K_{s}<17.9$ & ALL & 2715 & 1.05 & 1.80 & $0.305 \pm 0.025$ & 3.02 & $10.73 \pm 0.88$ & $12.5_{-2.9}^{+3.3}$ \\
\hline$R-K_{s}>5.0$ & $K_{s}<18.4$ & ALL & 6801 & 1.09 & 1.80 & $0.238 \pm 0.015$ & 3.86 & $9.63 \pm 0.61$ & $8.9_{-1.6}^{+1.7}$ \\
\hline$R-K_{s}>5.0$ & $K_{s}<18.9$ & ALL & 15013 & 1.12 & 1.80 & $0.161 \pm 0.008$ & 3.94 & $7.86 \pm 0.39$ & $4.7_{-0.7}^{+0.8}$ \\
\hline$R-K_{s}>5.0$ & $K_{s}<19.4$ & ALL & 28724 & 1.13 & 1.80 & $0.115 \pm 0.006$ & 7.03 & $6.49 \pm 0.34$ & $2.9_{-1.0}^{+1.5}$ \\
\hline$I-K_{s}>4.0$ & $K_{s}<17.9$ & ALL & 1314 & 1.08 & 1.80 & $0.225 \pm 0.035$ & 3.54 & $9.88 \pm 1.54$ & $9.7_{-3.9}^{+5.5}$ \\
\hline$I-K_{s}>4.0$ & $K_{s}<18.4$ & ALL & 3672 & 1.15 & 1.80 & $0.291 \pm 0.021$ & 3.94 & $10.89 \pm 0.79$ & $13.1_{-2.7}^{+3.1}$ \\
\hline$I-K_{s}>4.0$ & $K_{s}<18.9$ & ALL & 9829 & 1.18 & 1.80 & $0.205 \pm 0.011$ & 8.72 & $8.77 \pm 0.47$ & $6.7_{-1.1}^{+1.2}$ \\
\hline$I-K_{s}>4.0$ & $K_{s}<19.4$ & ALL & 22451 & 1.19 & 1.80 & $0.140 \pm 0.007$ & 11.85 & $7.10 \pm 0.36$ & $3.4_{-0.4}^{+0.6}$ \\
\hline
\end{tabular}

Note. Clustering results for samples with $N<500$ are not shown due to poor-quality statistics for our field area.

selected by the three ERO selection criteria. Quantitatively, the bulk $(>86 \%)$ of the $\mathrm{ERO}_{I K}$ sample is a sub-sample of both the $\mathrm{ERO}_{I 3.6}$ and $\mathrm{ERO}_{R K}$ samples. Of the $\mathrm{ERO}_{R K}$ sample, 78\% have $(I-[3.6])>5.0$; however, only $\approx 55 \%$ of the $\mathrm{ERO}_{I 3.6}$ sample have $\left(I-K_{s}\right)>4.0$ and $\left(R-K_{s}\right)>5.0$. The differences are likely due to the $\mathrm{ERO}_{13.6}$ having a greater surface density because at $3.6 \mu \mathrm{m}$ we are detecting rest-frame NIR emission, which is less obscured by dust. Thus, both the $\mathrm{ERO}_{R K}$ and $\mathrm{ERO}_{I K}$ samples are largely subsets of the $\mathrm{ERO}_{I 3.6}$ sample, but with different mean redshift. To determine whether there is a relationship between ERO clustering strength and luminosity, we separate each ERO sample into four [3.6] and $K_{s}$ magnitudelimited sub-samples as defined in Tables 1 and 3. Again, since all of our samples contain at least $\gtrsim 1000$ objects with photometric redshifts, the statistical uncertainties in the mean redshifts are negligible.

\subsection{ERO-type Separation}

At low redshift the clustering of passive galaxies differs from that of star-forming galaxies (e.g., Zehavi et al. 2010), but this may change with redshift (e.g., $z \gtrsim 1.3-2.0$; Cooper et al. 2007; Brodwin et al. 2008). There is no reason to expect passive and star-forming galaxies to occupy the same halos and have identical clustering properties. It is thus both interesting and necessary to separate star-forming EROs from passive EROs in order to determine if they show differing clustering properties. If star-forming EROs have distinctly different clustering properties from their passive counterparts, it would show that they are two distinct populations of galaxies, and it would mean that they are the progenitors of two very different populations of local universe galaxies. It would also mean that clustering studies of mixed ERO populations cannot be used to accurately link EROs to local galaxy populations.

To independently measure the clustering of passive and star-forming EROs, we use the wealth of data in the EGS to explore mid-IR color separation techniques using IRAC (Wilson et al. 2007) and MIPS (Stern et al. 2006) colors. These techniques are physically motivated by polycyclic aromatic hydrocarbon (PAH) emission from warm dust in star-forming regions. The ([3.6] - [8.0]) color is able to separate the ERO types as long as the strong PAH emission features lie in $8.0 \mu \mathrm{m}$ band, but this ceases to be true for $z \gtrsim 1$. Stern et al. (2006) showed that the difference in ([3.6] - [8.0]) color between a starforming ERO and a passive ERO can be very small for archetypal star-forming and passive EROs. Kong et al. (2009) also find that very high precision photometry is required to produce good agreement between ([3.6] - [8.0]) color and morphological ERO classifications. We tested the ([3.6] - [8.0]) color of EROs in the EGS using DEEP2 spectroscopic indicators. Although we do observe a tendency for passive EROs to have a bluer ([3.6] - [8.0]) color than star-forming ones, the two populations 


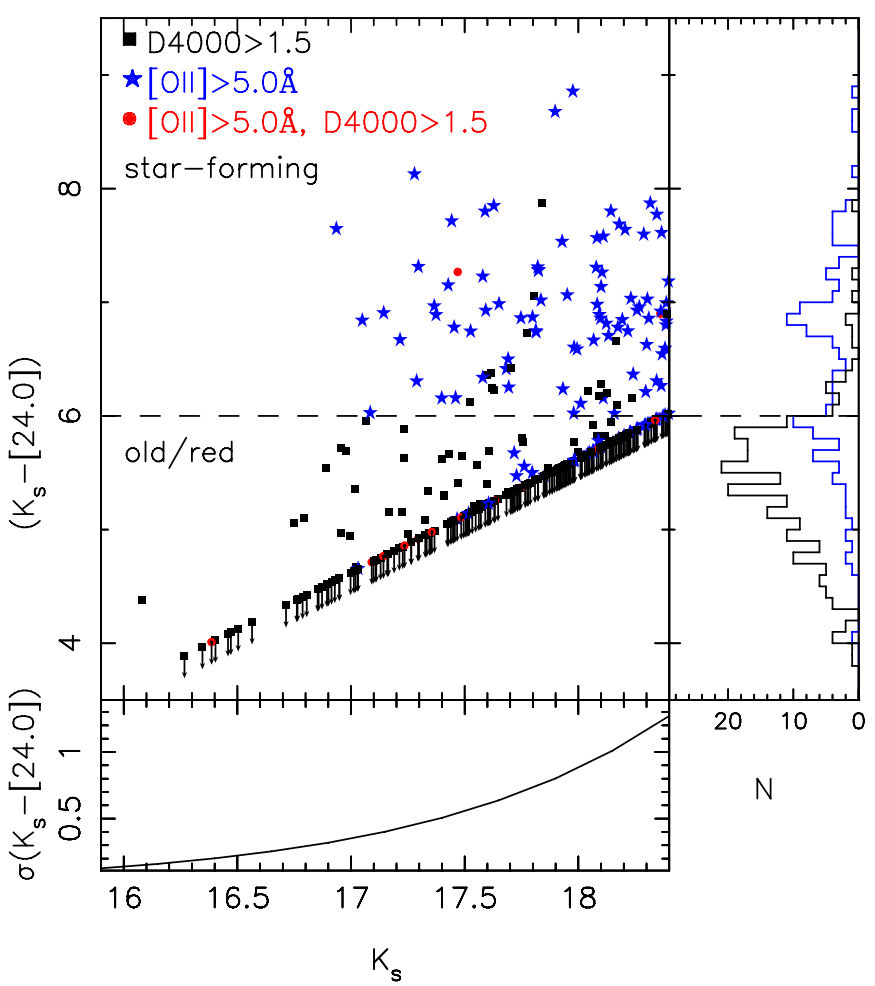

Figure 7. Galaxy-type separation using $\left(K_{s}-[24]\right)$ colors as a function of $K_{s}$ for all $0.6<z<1.5$ galaxies in the EGS. Objects with $f_{24}<80 \mu$ Jy are indicated by arrows. Sources are marked by whether they have D4000 $>1.5$, indicating an old stellar population (squares), $[\mathrm{O} \mathrm{II}]>5.0 \AA$, indicating star formation (stars), or both (circles). We empirically separate the populations at $\left(K_{s}-[24]\right)=6.0$. The FIDEL fluxes have been degraded in precision to match the noise and detection limit of the Boötes data. The histogram in the side panel shows the color distribution of D4000 $>1.5$ sources (black line) and $[\mathrm{O}$ II $]>5.0 \AA$ sources (blue line). The lower panel shows $\sigma\left(K_{s}-[24]\right)$ as a function of apparent magnitude based on a $24 \mu \mathrm{m}$ rms of $40 \mu \mathrm{Jy}$.

(A color version of this figure is available in the online journal.)

are not well separated and this technique is not suitable for this work.

Stern et al. (2006) showed that the $\left(K_{s}-[24]\right)$ color has a much broader color separation than the ([3.6] - [8.0]) color for archetypal star-forming and passive EROs. In Figure 7, $\left(K_{s}-\right.$ [24]) color versus $K_{s}$ magnitude is shown for $0.6<$ $z<1.5$ galaxies in the EGS that have $B_{w} R I K_{s}$, IRAC, $24 \mu \mathrm{m}$, and DEEP2 detections. We independently identify passive EROs using the DEEP2 D4000 spectroscopic indicator, which provides a measure of the strength of the $4000 \AA$ break. D4000 $>1.5$ is used to indicate an evolved stellar population. To independently identify the star-forming EROs, we use a DEEP2 rest-frame [O II] equivalent width $>5.0 \AA$ to identify galaxies with significant active star formation. Only DEEP2 spectroscopic indicators with $\mathrm{a} \geqslant 3 \sigma$ detection were used in this analysis.

The FIDEL $24 \mu \mathrm{m}$ catalog has a $3 \sigma$ depth of $30 \mu \mathrm{Jy}$, so we use this as the upper limit on any source without a $24 \mu \mathrm{m}$ detection. The Boötes field $24 \mu \mathrm{m}$ data (MAGES) have an rms of $40 \mu \mathrm{Jy}$ and thus a $2 \sigma$ depth of $80 \mu \mathrm{Jy}$. To determine how the noisier and shallower MAGES data will effect population separation criteria, in Figure 7 we have applied additional Gaussian scatter to the FIDEL data to mimic the rms noise level of MAGES. The two populations start to merge at $K_{s}=18.4$ and [3.6] $=17.5$, so the $2 \sigma$ detection limit of MAGES prevents us from separating the two populations at fainter magnitude.

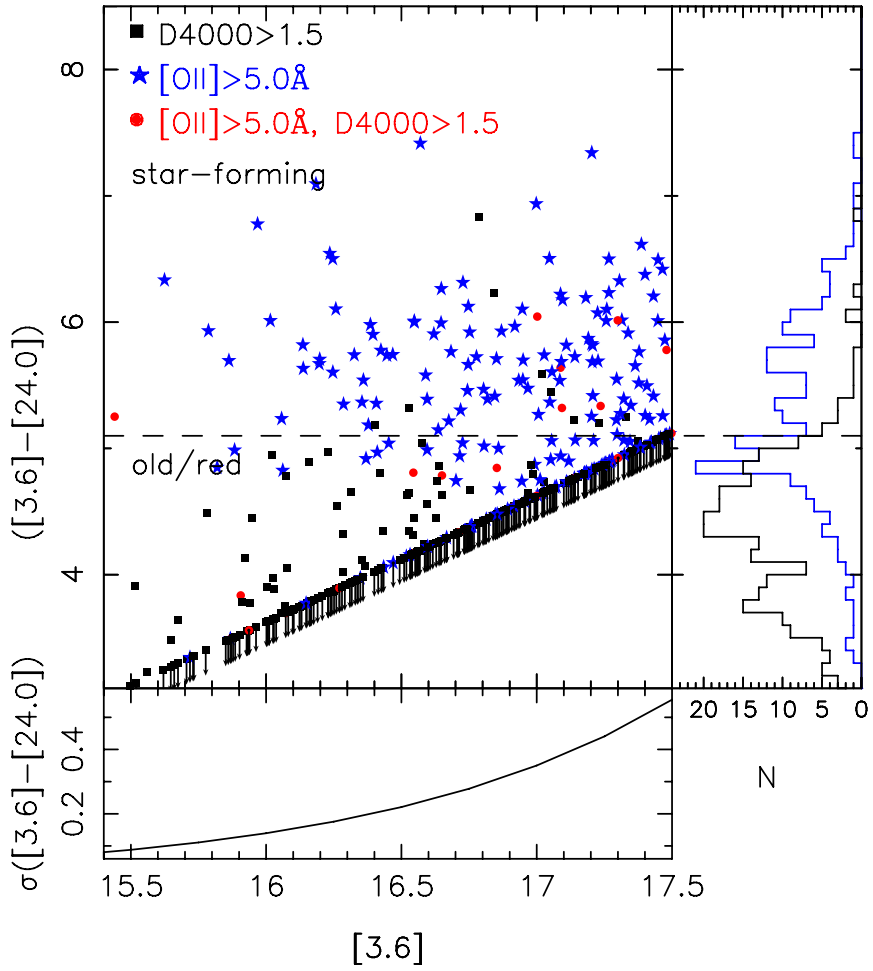

Figure 8. Galaxy-type separation using ([3.6] - [24]) colors as a function of [3.6] for all $0.6<z<1.5$ galaxies in the EGS. Objects with $f_{24}<80 \mu$ Jy are indicated by arrows. Sources are marked by whether they have D4000 $>1.5$, indicating an old stellar population (squares), $\left[\mathrm{O}_{\mathrm{II}}\right]>5.0 \AA$, indicating star formation (stars), or both (circles). We empirically separate the populations at $([3.6]-[24])=5.2$. The FIDEL fluxes have been degraded in precision to match the noise and detection limit of the Boötes data. The histogram in the side panel shows the color distribution of D4000 $>1.5$ sources (black line) and $\left[\mathrm{O}_{\mathrm{II}}\right]>5.0 \AA$ sources (blue line). The lower panel shows $\sigma([3.6]-[24])$ as a function of apparent magnitude based on a $24 \mu \mathrm{m}$ rms of $40 \mu \mathrm{Jy}$.

(A color version of this figure is available in the online journal.)

Since the $3.6 \mu \mathrm{m}$ imaging in SDWFS is significantly deeper than the NEWFIRM $K_{s}$ in Boötes, not every source with a $3.6 \mu \mathrm{m}$ detection will have a good $K_{s}$ detection. To achieve better separation for the $\mathrm{ERO}_{I 3.6}$ sample, we tested using the ([3.6] - [24]) color as a separation method. Figure 8 shows that this also works in a similar manner to the $\left(K_{s}-[24]\right)$ color. In both Figures 7 and 8 the EROs with strong D4000 and those with strong [O II] are well separated. This is further supported by the histograms in Figures 7 and 8, which show a bimodal distribution in color, but with some overlap, for these two spectral indicators. Some galaxies have both strong D4000 and $\left[\mathrm{O}_{\mathrm{II}}\right]$. For these objects we visually inspected the DEEP2 1D spectra to confirm the color classification and found that it accurately distinguished between passive and star-forming galaxies in at least $90 \%$ of cases.

We empirically place a discriminator between passive and star-forming EROs where the base of the strong D4000 distribution (seen in Figures 7 and 8) and the respective color for an object at the $24 \mu \mathrm{m}$ detection limit meet. Using a $2 \sigma$ detection limit for a MAGES source, we can use this separation technique out to $K_{s}=18.4$ and [3.6] $=17.5$, with discriminators at $\left(K_{s}-[24]\right)=6.0$ and $([3.6]-[24])=5.2$, respectively. We consider $2 \sigma$ a significant limit for the purposes of this work as we are interested in whether the source is emitting at $24 \mu \mathrm{m}$ rather than its specific flux. Since the placement of these discriminators is empirical, we varied the discriminators by \pm 0.2 


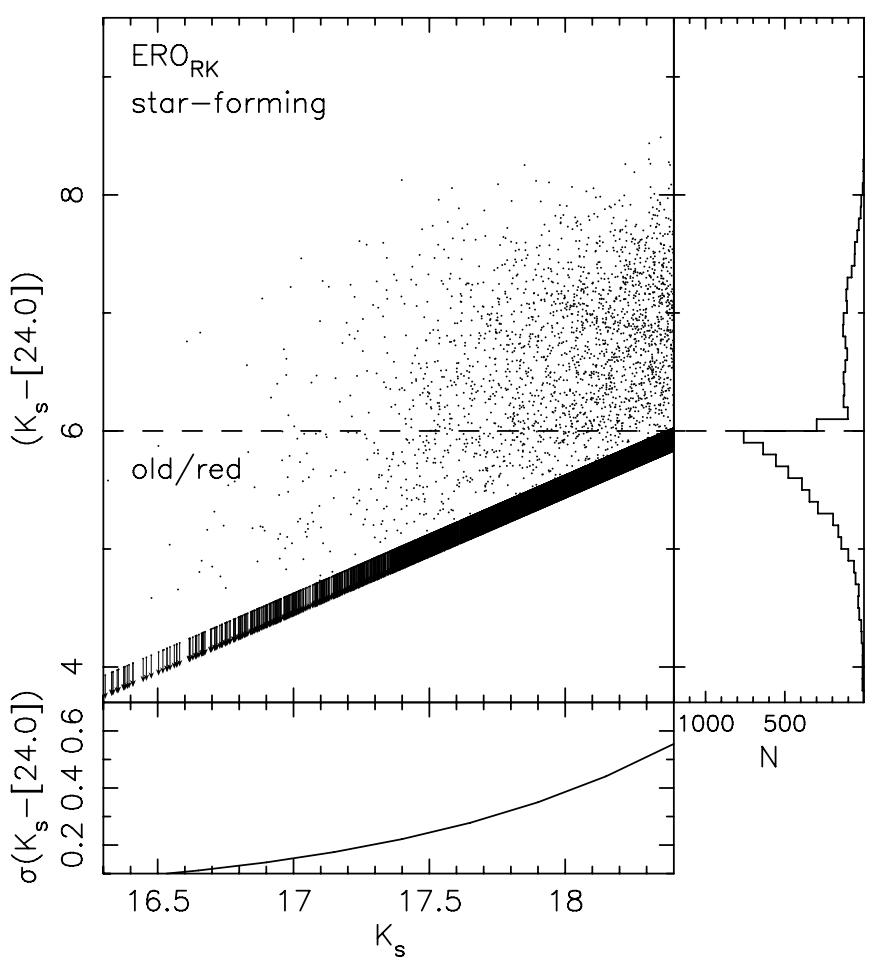

Figure 9. Separating star-forming and passive EROs using the $\left(K_{s}-[24]\right)$ color in the $\mathrm{ERO}_{R K}$ sample. We divide the populations at the dashed line $\left(K_{s}-[24]\right)=6.0$. Upper limits $f_{24 l i m}$ are marked with arrows. The histogram in the side panel shows the relative distribution in color space for Boötes objects down to $K_{s}<18.4$. The lower panel shows $\sigma\left(K_{s}-[24]\right)$ as a function of apparent magnitude based on a $24 \mu \mathrm{m}$ rms of $40 \mu \mathrm{Jy}$.

and found no significant change in the separated samples and final clustering results.

We define objects with $\left(K_{s}-[24]\right)>6.0$ and $([3.6]-[24])>$ 5.2 as star forming and those with $\left(K_{s}-[24]\right) \leqslant 6.0$ and $([3.6]-[24]) \leqslant 5.2$ as passive for $K_{s}<18.4$ and [3.6] $<17.5$, respectively. Details for the separated $\mathrm{ERO}_{R K}, \mathrm{ERO}_{I K}$, and ERO $_{I 3.6}$ samples are given in Tables 2 and 4, including the sample size, magnitude limit, $\bar{z}$, and relative fractions of the combined ERO samples to the same limiting magnitude. As a check, the archetypal star-forming ERO HR10 (Graham \& Dey 1996) with $\left(K_{s}-[24]\right)=7.61$ and $([3.6]-[24])=5.82$ (Stern et al. 2006) is clearly classified as star-forming, while the archetypal passive galaxy LBDS 53W091 (Stern et al. 2006) with $\left(K_{s}-[24]\right)<5.56$ and $([3.6]-[24])<4.13$ (assuming a $24 \mu \mathrm{m}$ flux upper limit of $40 \mu \mathrm{Jy}$ ) is clearly classified as passive.

There is some contamination on both sides of our color criteria, and the level of contamination increases for fainter sources in both Figures 7 and 8 . One possible physical reason for this is that PAH emission at observed $24 \mu \mathrm{m}$ varies with redshift. There is also rest-frame $10 \mu \mathrm{m}$ silicate absorption that would be observed at $24 \mu \mathrm{m}$ from a $z \approx 1.3 \mathrm{ERO}$. Contamination of this nature would therefore increase for fainter EROs since these are more likely to have higher redshifts.

Alternatively, EROs are probably not simply archetypal passive and star-forming galaxies. If we assume that the spectral energy distribution of a passive galaxy with little dust roughly follows a blackbody at long wavelengths, we can approximate the expected $24 \mu \mathrm{m}$ flux by the Rayleigh-Jeans tail. For LBDS $53 \mathrm{~W} 091$ with a $3.6 \mu \mathrm{m}$ flux of $35 \mu \mathrm{Jy}$, we expect an approximate $24 \mu \mathrm{m}$ flux of $0.02 \mu \mathrm{Jy}$ and so should have no $24 \mu \mathrm{m}$ detections for truly passive galaxies. However, there are objects

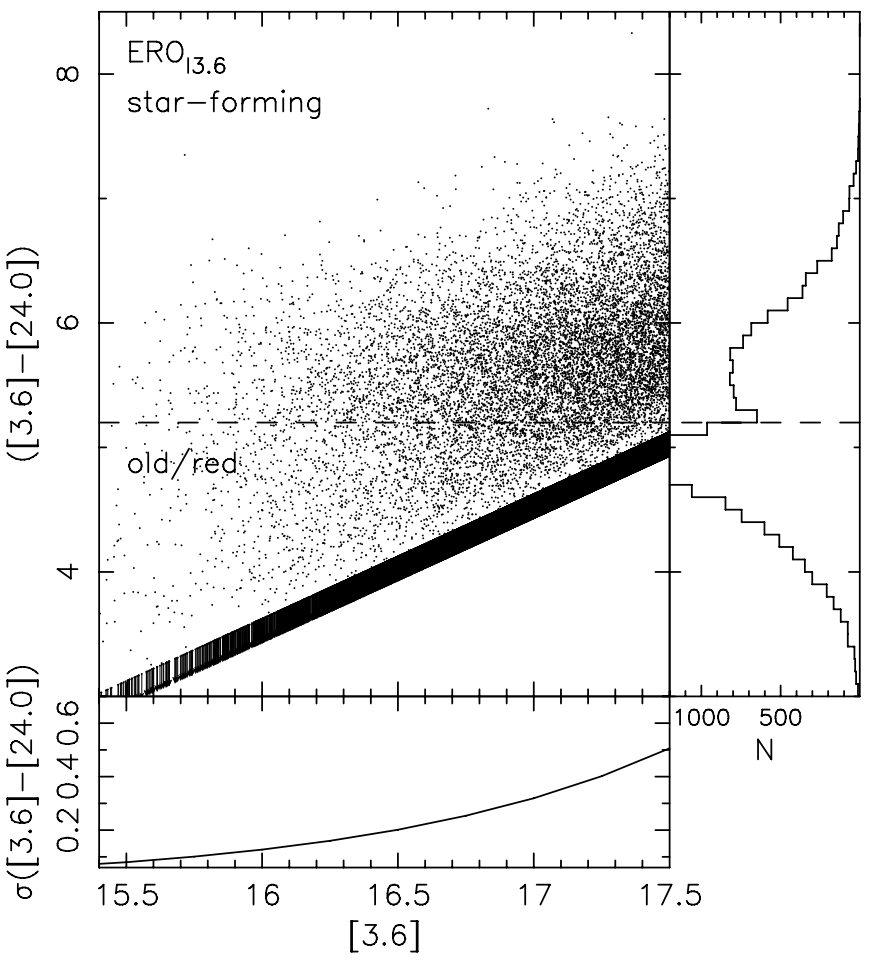

Figure 10. Separating star-forming and passive EROs using the ([3.6] - [24]) color in the $\mathrm{ERO}_{I 3.6}$ sample. We divide the populations at the dashed line $([3.6]-[24])=5.2$. Upper limits $f_{24 l i m}$ are marked with arrows. The histogram in the side panel shows the relative distribution in color space for Boötes objects down to [3.6] $<17.5$. The lower panel shows $\sigma([3.6]-[24])$ as a function of apparent magnitude based on a $24 \mu \mathrm{m} \mathrm{rms}$ of $40 \mu \mathrm{Jy}$.

with both a $24 \mu \mathrm{m}$ detection and strong D4000 in both Figures 7 and 8. The $\left(K_{s}-[24]\right)$ and ([3.6] - [24]) colors are effectively sampling the specific star formation rate (SSFR) of these galaxies. Therefore, these mixed cases are likely passive galaxies with some star formation and/or an AGN. To cross-check that this is the case, we further examine our cut against purely $24 \mu \mathrm{m}$ non-detected and detected samples, indicating SFR only (discussed in Section 4.3).

We show the application of these separation criteria to the Boötes field $\mathrm{ERO}_{R K}$ and $\mathrm{ERO}_{I 3.6}$ samples in Figures 9 and 10, respectively. The star-forming EROs form a locus that broadens toward fainter magnitudes. The broadening is largely an effect of the growth in the $\left(K_{s}-\right.$ [24]) and ([3.6] - [24]) uncertainties with magnitude, which we show in the lower panel of each figure. Despite the broadening, it is clear that the $\left(K_{s}-[24]\right)$ and ([3.6] - [24]) colors are finding distinct populations. This is clearly evident in the bimodal distribution in ([3.6] - [24]) color shown by the histogram in Figure 10. Figure 9 shows weaker evidence for this bi-modality in the $\left(K_{s}-[24]\right)$ color, which may be due to the reduced fraction of star-forming EROs in the $\mathrm{ERO}_{R K}$ sample (see Tables 2 and 4).

The relative fractions of star-forming and passive EROs vary between the different ERO selection criteria. At $K_{s}<18.4$, the $\mathrm{ERO}_{R K}$ sample shows a star-forming fraction of $30 \%$. Similarly, at $K_{s}<18.4$, the $\mathrm{ERO}_{I K}$ sample shows a star-forming fraction of $32 \%$. In contrast, at [3.6] $<17.5$, the $\mathrm{ERO}_{I 3.6}$ sample has a significantly higher star-forming fraction of $41 \%$. The star-forming fractions found for the $\mathrm{ERO}_{R K}$ and $\mathrm{ERO}_{I K}$ samples are consistent with previous studies (e.g., the fraction of peculiar galaxies; Conselice et al. 2008, $\left.\left(R-K_{S}\right)>5.3\right)$. The relative fraction of star-forming EROs in the $\mathrm{ERO}_{I 3.6}$ 
Table 2

Summary of Type-separated ERO Clustering Results

\begin{tabular}{|c|c|c|c|c|c|c|c|c|c|c|}
\hline $\begin{array}{l}\text { ERO } \\
\text { Cut }\end{array}$ & $\begin{array}{l}\text { Mag. } \\
\text { Limit }\end{array}$ & $\begin{array}{l}\text { ERO } \\
\text { Type }\end{array}$ & $N$ & $\begin{array}{c}\% \\
\text { Total }\end{array}$ & $\bar{z}$ & $\gamma$ & $w\left(1^{\prime}\right)$ & $\chi^{2} /$ dof & $\begin{array}{c}r_{0} \\
\left(h^{-1} \mathrm{Mpc}\right)\end{array}$ & $\begin{array}{c}M_{\text {halo }} \\
\left(\times 10^{12} M_{\odot}\right)\end{array}$ \\
\hline$I-[3.6]>5.0$ & {$[3.6]<16.5$} & $\mathrm{E}$ & 2281 & 71 & 1.11 & $1.92 \pm 0.09$ & $0.419 \pm 0.041$ & 5.91 & $10.01 \pm 1.79$ & $10.1_{-4.7}^{+6.6}$ \\
\hline$I-[3.6]>5.0$ & {$[3.6]<16.5$} & SF & 928 & 29 & 1.09 & $1.82 \pm 0.14$ & $0.232 \pm 0.045$ & 7.83 & $8.75 \pm 2.27$ & $6.6_{-4.1}^{+7.0}$ \\
\hline$I-[3.6]>5.0$ & {$[3.6]<17.0$} & $\mathrm{E}$ & 6183 & 65 & 1.14 & $2.00 \pm 0.07$ & $0.265 \pm 0.020$ & 4.17 & $7.73 \pm 1.05$ & $4.5_{-1.7}^{+2.1}$ \\
\hline$I-[3.6]>5.0$ & {$[3.6]<17.0$} & SF & 3354 & 35 & 1.14 & $1.83 \pm 0.10$ & $0.139 \pm 0.016$ & 6.53 & $7.05 \pm 1.45$ & $3.3_{-1.7}^{+2.7}$ \\
\hline$I-[3.6]>5.0$ & {$[3.6]<17.5$} & $\mathrm{E}$ & 12905 & 59 & 1.16 & $1.95 \pm 0.06$ & $0.185 \pm 0.013$ & 2.98 & $6.84 \pm 0.88$ & $3.0_{-1.1}^{+1.4}$ \\
\hline$I-[3.6]>5.0$ & {$[3.6]<17.5$} & $\mathrm{SF}$ & 8895 & 41 & 1.16 & $1.87 \pm 0.09$ & $0.091 \pm 0.008$ & 5.52 & $5.52 \pm 1.02$ & $1.5_{-0.7}^{+1.1}$ \\
\hline$R-K_{s}>5.0$ & $K_{s}<17.9$ & $\mathrm{E}$ & 2213 & 82 & 1.04 & $1.89 \pm 0.08$ & $0.335 \pm 0.033$ & 3.02 & $10.36 \pm 1.87$ & $11.2_{-4.9}^{+7.5}$ \\
\hline$R-K_{s}>5.0$ & $K_{s}<17.9$ & $\mathrm{SF}$ & 502 & 18 & 1.06 & $2.04 \pm 0.17$ & $0.401 \pm 0.075$ & 4.50 & $9.20 \pm 2.70$ & $7.7_{-5.2}^{+8.6}$ \\
\hline$R-K_{s}>5.0$ & $K_{s}<18.4$ & $\mathrm{E}$ & 4744 & 70 & 1.08 & $1.85 \pm 0.06$ & $0.260 \pm 0.022$ & 5.56 & $9.52 \pm 1.50$ & $8.6_{-3.5}^{+5.0}$ \\
\hline$R-K_{s}>5.0$ & $K_{s}<18.4$ & $\mathrm{SF}$ & 2057 & 30 & 1.11 & $1.79 \pm 0.09$ & $0.262 \pm 0.031$ & 1.97 & $10.34 \pm 2.23$ & $11.1_{-5.5}^{+9.3}$ \\
\hline$I-K_{s}>4.0$ & $K_{s}<17.9$ & $\mathrm{E}$ & 1114 & 85 & 1.07 & $1.73 \pm 0.12$ & $0.247 \pm 0.042$ & 2.37 & $11.37 \pm 2.90$ & $14.9_{-8.8}^{+15.2}$ \\
\hline$I-K_{s}>4.0$ & $K_{s}<17.9$ & SF & 200 & 15 & 1.15 & $\ldots$ & $\ldots$ & $\ldots$ & $\ldots$ & $\ldots$ \\
\hline$I-K_{s}>4.0$ & $K_{s}<18.4$ & $\mathrm{E}$ & 2481 & 68 & 1.13 & $1.78 \pm 0.09$ & $0.313 \pm 0.033$ & 5.14 & $11.60 \pm 2.46$ & $15.9_{-7.8}^{+12.9}$ \\
\hline$I-K_{s}>4.0$ & $K_{s}<18.4$ & SF & 1191 & 32 & 1.18 & $1.61 \pm 0.10$ & $0.332 \pm 0.051$ & 3.27 & $13.95 \pm 3.33$ & $28.1_{-15.7}^{+26.2}$ \\
\hline$I-[3.6]>5.0$ & {$[3.6]<16.5$} & $\mathrm{E}$ & 2281 & 71 & 1.11 & 1.80 & $0.456 \pm 0.035$ & 6.00 & $11.82 \pm 0.91$ & $16.9_{-3.7}^{+4.3}$ \\
\hline$I-[3.6]>5.0$ & {$[3.6]<16.5$} & $\mathrm{SF}$ & 928 & 29 & 1.09 & 1.80 & $0.231 \pm 0.045$ & 7.76 & $8.89 \pm 1.73$ & $6.9_{-3.4}^{+5.2}$ \\
\hline$I-[3.6]>5.0$ & {$[3.6]<17.0$} & $\mathrm{E}$ & 6183 & 65 & 1.14 & 1.80 & $0.315 \pm 0.019$ & 4.86 & $10.12 \pm 0.61$ & $10.4_{-1.8}^{+2.0}$ \\
\hline$I-[3.6]>5.0$ & {$[3.6]<17.0$} & SF & 3354 & 35 & 1.14 & 1.80 & $0.141 \pm 0.016$ & 6.38 & $7.27 \pm 0.83$ & $3.7_{-1.2}^{+1.4}$ \\
\hline$I-[3.6]>5.0$ & {$[3.6]<17.5$} & $\mathrm{E}$ & 12905 & 59 & 1.16 & 1.80 & $0.212 \pm 0.011$ & 3.43 & $8.29 \pm 0.43$ & $5.6_{-0.9}^{+1.0}$ \\
\hline$I-[3.6]>5.0$ & {$[3.6]<17.5$} & $\mathrm{SF}$ & 8895 & 41 & 1.16 & 1.80 & $0.095 \pm 0.007$ & 5.39 & $5.91 \pm 0.44$ & $1.9_{-0.4}^{+0.5}$ \\
\hline$R-K_{s}>5.0$ & $K_{s}<17.9$ & $\mathrm{E}$ & 2213 & 82 & 1.04 & 1.80 & $0.357 \pm 0.030$ & 3.01 & $11.77 \pm 0.99$ & $16.6_{-4.0}^{+4.7}$ \\
\hline$R-K_{s}>5.0$ & $K_{s}<17.9$ & $\mathrm{SF}$ & 502 & 18 & 1.06 & 1.80 & $0.400 \pm 0.080$ & 4.42 & $11.69 \pm 2.34$ & $16.3_{-8.2}^{+12.1}$ \\
\hline$R-K_{s}>5.0$ & $K_{s}<18.4$ & $\mathrm{E}$ & 4744 & 70 & 1.08 & 1.80 & $0.271 \pm 0.019$ & 5.41 & $10.23 \pm 0.72$ & $10.8_{-2.1}^{+2.5}$ \\
\hline$R-K_{s}>5.0$ & $K_{s}<18.4$ & $\mathrm{SF}$ & 2057 & 30 & 1.11 & 1.80 & $0.261 \pm 0.027$ & 2.00 & $10.21 \pm 1.06$ & $10.7_{-3.1}^{+3.8}$ \\
\hline$I-K_{s}>4.0$ & $K_{s}<17.9$ & $\mathrm{E}$ & 1114 & 85 & 1.07 & 1.80 & $0.246 \pm 0.040$ & 2.52 & $10.56 \pm 1.72$ & $11.9_{-5.1}^{+7.1}$ \\
\hline$I-K_{s}>4.0$ & $K_{s}<17.9$ & SF & 200 & 15 & 1.15 & $\ldots$ & $\ldots$ & $\cdots$ & $\ldots$ & $\ldots$ \\
\hline$I-K_{s}>4.0$ & $K_{s}<18.4$ & $\mathrm{E}$ & 2481 & 68 & 1.13 & 1.80 & $0.309 \pm 0.027$ & 5.20 & $11.29 \pm 0.99$ & $14.6_{-3.6}^{+4.1}$ \\
\hline$I-K_{s}>4.0$ & $K_{s}<18.4$ & $\mathrm{SF}$ & 1191 & 32 & 1.18 & 1.80 & $0.321 \pm 0.042$ & 4.64 & $11.17 \pm 1.46$ & $14.2_{-4.9}^{+6.6}$ \\
\hline
\end{tabular}

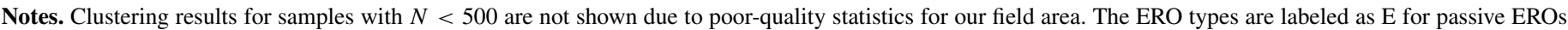
and SF for star-forming EROs. \% total is the fraction of combined EROs in a particular ERO-type sub-population.

sample is consistent with that found by Kong et al. (2009) using $(i-K)>3.96$. Moustakas et al. (2004) used an $\left(R-K_{s}\right)>5.0$ ERO cut and found a passive ERO fraction of only $33 \%-44 \%$, but this study went to a much fainter $K_{s}<22$ flux limit. We observe in all three ERO samples an increase of star-forming ERO fraction with increasing apparent magnitude (see Tables 2 and 4 and Figures 7-10), and this is consistent with the fraction of star-forming EROs increasing with decreasing luminosity and stellar mass (e.g., Moustakas et al. 2004; Conselice et al. 2008; Kong et al. 2009; Kim et al. 2011).

\section{ANALYSIS}

In this section, we analyze the angular and spatial clustering of our ERO samples. Using these methods, we place the EROs in an evolutionary sequence and make links to local galaxy populations. In Section 4.1, we study the angular clustering of each ERO sample as a function of limiting magnitude and ERO color and selection criterion. The three ERO samples studied, $\mathrm{ERO}_{R K}, \mathrm{ERO}_{I K}$, and $\mathrm{ERO}_{I 3.6}$, allow us to explore this to some degree across different redshift intervals. We build model redshift distributions in Section 4.2 and discuss the effects and limitations of the photometric redshifts used to generate them. We combine the angular clustering and the redshift distribution models to estimate the spatial correlation function in Section 4.3. We also analyze the spatial correlation function as a function of limiting magnitude and ERO color and selection criterion. We use the clustering of EROs and dark matter halos to connect EROs to other galaxy populations across redshift in Section 4.4. Finally, we estimate the host dark matter halo masses of our EROs in Section 4.5.

\subsection{The Angular Correlation Function}

We measured the angular correlation function for each magnitude-limited sample using the Landy \& Szalay (1993) estimator,

$$
\hat{w}(\theta)=\frac{\mathrm{DD}-2 \mathrm{DR}+\mathrm{RR}}{\mathrm{RR}},
$$

where DD, DR, and RR are the number of data-data, data-random, and random-random galaxy pairs at angular separation $\theta \pm \delta \theta / 2$, respectively. Pairs were counted in logarithmically spaced angular bins with $\Delta \log _{10} \theta=0.25$. Since any survey area represents a subset of the universe, estimators such as Equation (1) are subject to an integral constraint (Groth \& Peebles 1977),

$$
\iint \hat{w}\left(\theta_{12}\right) d \Omega_{1} d \Omega_{2} \simeq 0,
$$


Table 3

Summary of Combined ERO Clustering Results (ONLY Galaxies with $z_{\text {phot }}$ Information)

\begin{tabular}{|c|c|c|c|c|c|c|c|c|c|}
\hline ERO Cut & Mag. Limit & ERO Type & $N$ & $\bar{z}$ & $\gamma$ & $w\left(1^{\prime}\right)$ & $\chi^{2} /$ dof & $\begin{array}{c}r_{0} \\
\left(h^{-1} \mathrm{Mpc}\right)\end{array}$ & $\begin{array}{c}M_{\text {halo }} \\
\left(\times 10^{12} M_{\odot}\right)\end{array}$ \\
\hline$I-[3.6]>5.0$ & {$[3.6]<16.5$} & ALL & 2539 & 1.11 & $1.89 \pm 0.07$ & $0.357 \pm 0.033$ & 3.25 & $9.88 \pm 1.68$ & $9.7_{-3.8}^{+6.1}$ \\
\hline$I-[3.6]>5.0$ & {$[3.6]<17.0$} & ALL & 7464 & 1.14 & $1.88 \pm 0.05$ & $0.254 \pm 0.019$ & 1.88 & $8.74 \pm 1.21$ & $6.6_{-2.3}^{+3.3}$ \\
\hline$I-[3.6]>5.0$ & {$[3.6]<17.5$} & ALL & 15380 & 1.16 & $1.76 \pm 0.06$ & $0.198 \pm 0.016$ & 1.56 & $8.64 \pm 1.22$ & $6.3_{-2.4}^{+2.9}$ \\
\hline$I-[3.6]>5.0$ & {$[3.6]<18.0$} & ALL & 22754 & 1.17 & $1.73 \pm 0.05$ & $0.158 \pm 0.012$ & 2.24 & $7.89 \pm 1.18$ & $4.8_{-1.7}^{+2.6}$ \\
\hline$I-[3.6]>5.0$ & {$[3.6]<18.5$} & ALL & 25703 & 1.17 & $1.76 \pm 0.05$ & $0.145 \pm 0.011$ & 2.80 & $7.39 \pm 1.04$ & $3.9_{-1.4}^{+2.0}$ \\
\hline$R-K_{s}>5.0$ & $K_{s}<17.9$ & ALL & 2155 & 1.05 & $1.68 \pm 0.07$ & $0.402 \pm 0.043$ & 3.68 & $14.36 \pm 2.65$ & $30.7_{-13.8}^{+21.1}$ \\
\hline$R-K_{s}>5.0$ & $K_{s}<18.4$ & ALL & 5395 & 1.09 & $1.76 \pm 0.06$ & $0.312 \pm 0.027$ & 1.94 & $11.67 \pm 1.85$ & $16.2_{-6.5}^{+9.3}$ \\
\hline$R-K_{s}>5.0$ & $K_{s}<18.9$ & ALL & 10795 & 1.12 & $1.69 \pm 0.05$ & $0.239 \pm 0.021$ & 1.87 & $10.90 \pm 1.85$ & $13.1_{-5.1}^{+8.2}$ \\
\hline$R-K_{s}>5.0$ & $K_{s}<19.4$ & ALL & 15209 & 1.13 & $1.70 \pm 0.06$ & $0.205 \pm 0.017$ & 1.37 & $9.80 \pm 1.45$ & $9.4_{-3.7}^{+5.0}$ \\
\hline$I-K_{s}>4.0$ & $K_{s}<17.9$ & ALL & 940 & 1.08 & $1.90 \pm 0.13$ & $0.290 \pm 0.046$ & 1.96 & $10.29 \pm 2.48$ & $11.0_{-6.4}^{+10.3}$ \\
\hline$I-K_{s}>4.0$ & $K_{s}<18.4$ & ALL & 2653 & 1.15 & $1.80 \pm 0.07$ & $0.353 \pm 0.034$ & 2.47 & $12.12 \pm 2.22$ & $18.2_{-7.8}^{+12.4}$ \\
\hline$I-K_{s}>4.0$ & $K_{s}<18.9$ & ALL & 6133 & 1.18 & $1.73 \pm 0.06$ & $0.260 \pm 0.023$ & 3.09 & $10.71 \pm 1.83$ & $12.4_{-5.2}^{+7.8}$ \\
\hline$I-K_{s}>4.0$ & $K_{s}<19.4$ & ALL & 8988 & 1.19 & $1.75 \pm 0.06$ & $0.217 \pm 0.018$ & 1.49 & $9.48 \pm 1.56$ & $8.5_{-3.2}^{+5.2}$ \\
\hline$I-[3.6]>5.0$ & {$[3.6]<16.5$} & ALL & 2539 & 1.11 & 1.80 & $0.381 \pm 0.030$ & 3.27 & $11.19 \pm 0.88$ & $14.2_{-3.2}^{+3.6}$ \\
\hline$I-[3.6]>5.0$ & {$[3.6]<17.0$} & ALL & 7464 & 1.14 & 1.80 & $0.273 \pm 0.016$ & 1.91 & $9.79 \pm 0.57$ & $9.4_{-1.6}^{+1.8}$ \\
\hline$I-[3.6]>5.0$ & {$[3.6]<17.5$} & ALL & 15380 & 1.16 & 1.80 & $0.190 \pm 0.010$ & 1.75 & $8.16 \pm 0.43$ & $5.3_{-0.8}^{+0.8}$ \\
\hline$I-[3.6]>5.0$ & {$[3.6]<18.0$} & ALL & 22754 & 1.17 & 1.80 & $0.147 \pm 0.007$ & 2.79 & $7.17 \pm 0.34$ & $3.5_{-0.5}^{+0.6}$ \\
\hline$I-[3.6]>5.0$ & {$[3.6]<18.5$} & ALL & 25703 & 1.17 & 1.80 & $0.139 \pm 0.006$ & 3.07 & $7.00 \pm 0.30$ & $3.2_{-0.4}^{+0.5}$ \\
\hline$R-K_{s}>5.0$ & $K_{s}<17.9$ & ALL & 2155 & 1.05 & 1.80 & $0.364 \pm 0.031$ & 4.32 & $11.84 \pm 1.01$ & $16.9_{-4.0}^{+4.9}$ \\
\hline$R-K_{s}>5.0$ & $K_{s}<18.4$ & ALL & 5395 & 1.09 & 1.80 & $0.301 \pm 0.019$ & 2.12 & $10.97 \pm 0.69$ & $13.4_{-2.5}^{+2.8}$ \\
\hline$R-K_{s}>5.0$ & $K_{s}<18.9$ & ALL & 10795 & 1.12 & 1.80 & $0.214 \pm 0.012$ & 2.86 & $9.21 \pm 0.52$ & $7.7_{-1.3}^{+1.3}$ \\
\hline$R-K_{s}>5.0$ & $K_{s}<19.4$ & ALL & 15209 & 1.13 & 1.80 & $0.185 \pm 0.010$ & 2.18 & $8.45 \pm 0.46$ & $6.6_{-2.3}^{+3.3}$ \\
\hline$I-K_{s}>4.0$ & $K_{s}<17.9$ & ALL & 940 & 1.08 & 1.80 & $0.297 \pm 0.048$ & 2.00 & $11.53 \pm 1.86$ & $15.6_{-6.6}^{+9.2}$ \\
\hline$I-K_{s}>4.0$ & $K_{s}<18.4$ & ALL & 2653 & 1.15 & 1.80 & $0.353 \pm 0.028$ & 2.47 & $12.12 \pm 0.96$ & $18.2_{-4.0}^{+4.8}$ \\
\hline$I-K_{s}>4.0$ & $K_{s}<18.9$ & ALL & 6133 & 1.18 & 1.80 & $0.243 \pm 0.016$ & 3.43 & $9.64 \pm 0.63$ & $9.0_{-1.6}^{+2.0}$ \\
\hline$I-K_{s}>4.0$ & $K_{s}<19.4$ & ALL & 8988 & 1.19 & 1.80 & $0.207 \pm 0.012$ & 1.73 & $8.83 \pm 0.51$ & $6.8_{-1.2}^{+1.2}$ \\
\hline
\end{tabular}

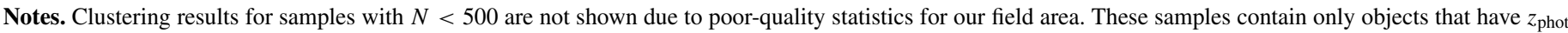
information.

where $\theta_{12}$ is the angular separation between solid angle elements $d \Omega_{1}$ and $d \Omega_{2}$. Since Equation (1) is normalized by itself, the integral constraint leads to a systematic underestimate of $\hat{w}(\theta)$, which is corrected by the addition of the variance term,

$$
\sigma^{2}=\frac{1}{\Omega^{2}} \iint \hat{w}\left(\theta_{12}\right) d \Omega_{1} d \Omega_{2} .
$$

This corrects for the contribution to clustering from the variance in number counts over the finite survey area $\Omega$. We estimate the uncertainty in each angular correlation function bin using a modification to the Gaussian approximation to the covariance matrix discussed in Brown et al. (2008). Our measured $w\left(1^{\prime}\right)$ values are well below unity, so we consider our uncertainty estimates valid to first order.

The angular correlation functions for the $\mathrm{ERO}_{R K} K_{s}<18.4$, $\mathrm{ERO}_{I K} K_{s}<18.4$, and $\mathrm{ERO}_{I 3.6}[3.6]<17.0$ samples are presented in Figure 11. Each of these ERO samples shows similar clustering statistics, which is an indication that similar galaxy populations are being included by each criterion. We then fit the angular correlation function for each sample using $\chi^{2}$ statistics including the full covariance matrix for the uncertainties and the functional form

$$
w(\theta)=w\left(1^{\prime}\right)\left(\frac{\theta}{1^{\prime}}\right)^{1-\gamma} .
$$

Many studies use a fixed value for $\gamma$ (e.g., Firth et al. 2002; Roche et al. 2002; Brown et al. 2005; Kong et al. 2009; Kim et al. 2011) because they have insufficient data to constrain $\gamma$. With our larger sample sizes $(N \gtrsim 2000)$, we are able to leave the power-law $\gamma$ as a free parameter. To better compare with the prior literature and for our smaller samples with $1000 \lesssim N<2000$, we also fit the correlation function with a fixed $\gamma=1.80$ (e.g., Kong et al. 2009; Kim et al. 2011). It should be noted that due to our field size, samples with $N<500$ do not produce reliable correlation functions and have been removed from our final results.

In many cases the reduced $\chi^{2}$ for our fits are distinctly greater than unity. We consider this an indication that a simple power law is not an adequate description of galaxy angular correlation functions. Some studies have tried to compensate for this with a double power law (e.g., Kim et al. 2011). However, this is again an empirical approximation and has no physical basis. For a robust analysis of galaxy correlation we must consider a more complex model of galaxy clustering such as galaxy-dark matter halo models (e.g., the Halo Occupation Distribution; Seljak 2000), which we will address in a future paper.

The angular clustering of EROs is strongly dependent on limiting magnitude. As shown in Figure 12, the brightest sample for each ERO criterion has a $w\left(1^{\prime}\right)$ clustering amplitude that is a factor of $\approx 3$ stronger than the faintest sample (as before, we take $K_{s} \approx[3.6]+1.4$, based on the average of $K_{s}-[3.6]$ 


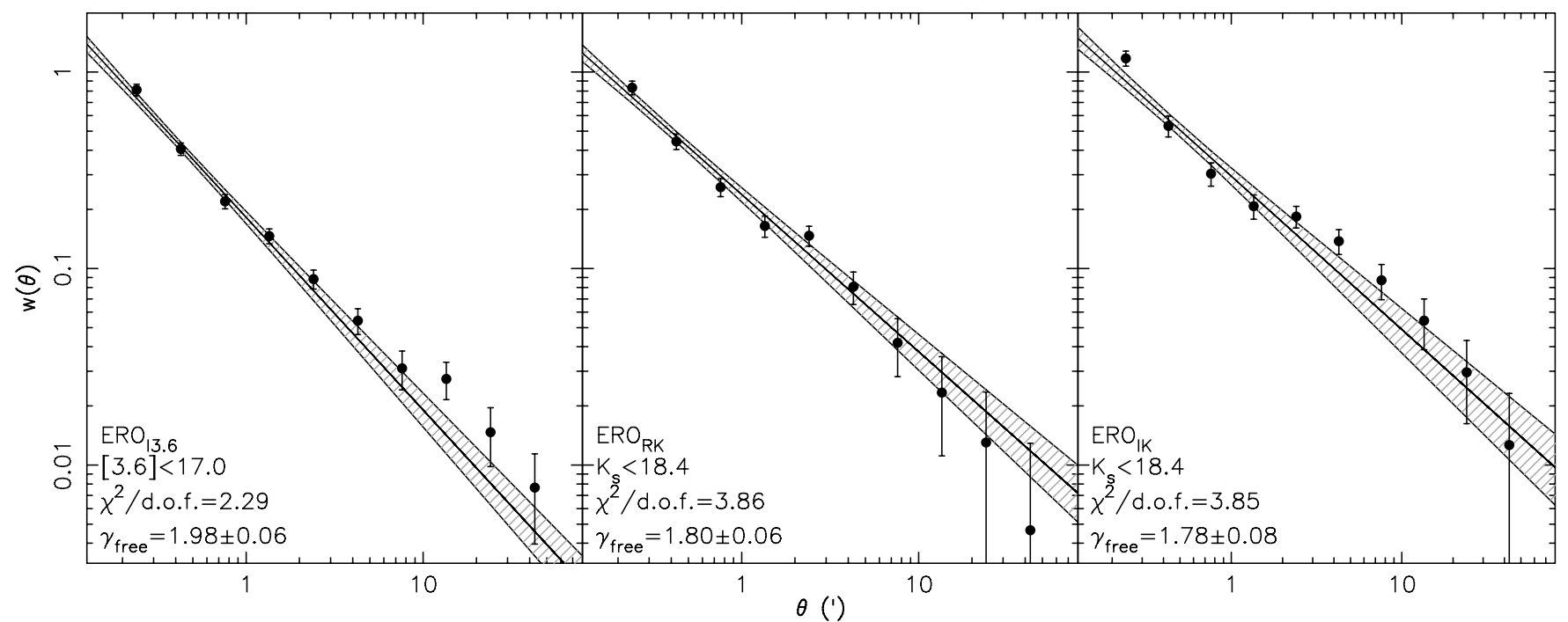

Figure 11. Angular correlation functions $w(\theta)$. From left to right we show $w(\theta)$ for the $\mathrm{ERO}_{I 3.6}[3.6]<17.0, \mathrm{ERO}_{R K} K_{s}<18.4$, and $\mathrm{ERO}_{I K} K_{s}<18.4$ samples. We also show the reduced $\chi^{2}$ and fitted power-law $\gamma$ for each fit. These three samples are selected using different ERO criteria but show very similar clustering statistics. This suggests that similar populations are being selected by all three ERO criteria.

Table 4

Summary of Type-separated ERO Clustering Results (ONLY Galaxies with $z_{\text {phot }}$ Information)

\begin{tabular}{|c|c|c|c|c|c|c|c|c|c|c|}
\hline $\begin{array}{l}\text { ERO } \\
\text { Cut }\end{array}$ & $\begin{array}{l}\text { Mag. } \\
\text { Limit }\end{array}$ & $\begin{array}{l}\text { ERO } \\
\text { Type }\end{array}$ & $N$ & $\begin{array}{c}\% \\
\text { Total }\end{array}$ & $\bar{z}$ & $\gamma$ & $w\left(1^{\prime}\right)$ & $\chi^{2} / \mathrm{dof}$ & $\begin{array}{c}r_{0} \\
\left(h^{-1} \mathrm{Mpc}\right)\end{array}$ & $\begin{array}{c}M_{\text {halo }} \\
\left(\times 10^{12} M_{\odot}\right)\end{array}$ \\
\hline$I-[3.6]>5.0$ & {$[3.6]<16.5$} & $\mathrm{E}$ & 1849 & 73 & 1.11 & $1.98 \pm 0.08$ & $0.500 \pm 0.045$ & 2.95 & $10.34 \pm 1.79$ & $11.1_{-4.5}^{+7.1}$ \\
\hline$I-[3.6]>5.0$ & {$[3.6]<16.5$} & SF & 690 & 27 & 1.09 & $1.57 \pm 0.09$ & $0.258 \pm 0.063$ & 6.83 & $11.88 \pm 3.20$ & $17.1_{-10.5}^{+18.6}$ \\
\hline$I-[3.6]>5.0$ & {$[3.6]<17.0$} & $\mathrm{E}$ & 5003 & 67 & 1.14 & $2.01 \pm 0.06$ & $0.330 \pm 0.024$ & 2.55 & $8.56 \pm 1.11$ & $6.1_{-2.2}^{+2.7}$ \\
\hline$I-[3.6]>5.0$ & {$[3.6]<17.0$} & $\mathrm{SF}$ & 2461 & 33 & 1.14 & $1.63 \pm 0.09$ & $0.188 \pm 0.026$ & 5.68 & $9.95 \pm 2.17$ & $9.9_{-5.1}^{+8.3}$ \\
\hline$I-[3.6]>5.0$ & {$[3.6]<17.5$} & $\mathrm{E}$ & 9697 & 63 & 1.16 & $1.90 \pm 0.05$ & $0.249 \pm 0.018$ & 1.28 & $8.30 \pm 1.14$ & $5.6_{-1.8}^{+2.8}$ \\
\hline$I-[3.6]>5.0$ & {$[3.6]<17.5$} & $\mathrm{SF}$ & 5683 & 37 & 1.16 & $1.88 \pm 0.08$ & $0.142 \pm 0.014$ & 7.01 & $6.95 \pm 1.33$ & $3.2_{-1.5}^{+2.4}$ \\
\hline$R-K_{s}>5.0$ & $K_{s}<17.9$ & $\mathrm{E}$ & 1761 & 82 & 1.04 & $1.92 \pm 0.08$ & $0.401 \pm 0.039$ & 3.49 & $11.04 \pm 1.94$ & $13.6_{-5.9}^{+8.9}$ \\
\hline$R-K_{s}>5.0$ & $K_{s}<17.9$ & $\mathrm{SF}$ & 394 & 18 & 1.06 & $\ldots$ & $\ldots$ & $\ldots$ & $\ldots$ & $=-3.0$ \\
\hline$R-K_{s}>5.0$ & $K_{s}<18.4$ & $\mathrm{E}$ & 3804 & 71 & 1.08 & $1.89 \pm 0.07$ & $0.327 \pm 0.028$ & 3.15 & $10.35 \pm 1.56$ & $11.2_{-4.5}^{+5.9}$ \\
\hline$R-K_{s}>5.0$ & $K_{s}<18.4$ & $\mathrm{SF}$ & 1591 & 29 & 1.11 & $1.61 \pm 0.08$ & $0.306 \pm 0.042$ & 2.36 & $13.71 \pm 3.09$ & $26.7_{-13.6}^{+23.2}$ \\
\hline$I-K_{s}>4.0$ & $K_{s}<17.9$ & $\mathrm{E}$ & 802 & 85 & 1.07 & $2.11 \pm 0.15$ & $0.318 \pm 0.051$ & 1.51 & $8.98 \pm 2.39$ & $7.2_{-4.5}^{+7.6}$ \\
\hline$I-K_{s}>4.0$ & $K_{s}<17.9$ & $\mathrm{SF}$ & 138 & 15 & 1.15 & $\ldots$ & $\ldots$ & $\ldots$ & $\ldots$ & \\
\hline$I-K_{s}>4.0$ & $K_{s}<18.4$ & $\mathrm{E}$ & 1810 & 68 & 1.13 & $1.96 \pm 0.10$ & $0.380 \pm 0.039$ & 3.30 & $10.74 \pm 2.01$ & $12.5_{-6.0}^{+8.3}$ \\
\hline$I-K_{s}>4.0$ & $K_{s}<18.4$ & $\mathrm{SF}$ & 843 & 32 & 1.18 & $1.69 \pm 0.10$ & $0.377 \pm 0.061$ & 2.69 & $13.77 \pm 3.29$ & $27.0_{-14.6}^{+25.3}$ \\
\hline$I-[3.6]>5.0$ & {$[3.6]<16.5$} & E & 1849 & 73 & 1.11 & 1.80 & $0.568 \pm 0.045$ & 3.63 & $13.36 \pm 1.06$ & $24.6_{-5.4}^{+6.5}$ \\
\hline$I-[3.6]>5.0$ & {$[3.6]<16.5$} & $\mathrm{SF}$ & 690 & 27 & 1.09 & 1.80 & $0.249 \pm 0.056$ & 8.54 & $9.27 \pm 2.08$ & $7.9_{-4.4}^{+7.0}$ \\
\hline$I-[3.6]>5.0$ & {$[3.6]<17.0$} & $\mathrm{E}$ & 5003 & 67 & 1.14 & 1.80 & $0.393 \pm 0.025$ & 3.49 & $11.45 \pm 0.73$ & $15.3_{-2.7}^{+3.2}$ \\
\hline$I-[3.6]>5.0$ & {$[3.6]<17.0$} & $\mathrm{SF}$ & 2461 & 33 & 1.14 & 1.80 & $0.176 \pm 0.021$ & 6.78 & $8.22 \pm 0.98$ & $5.4_{-1.8}^{+2.3}$ \\
\hline$I-[3.6]>5.0$ & {$[3.6]<17.5$} & $\mathrm{E}$ & 9697 & 63 & 1.16 & 1.80 & $0.273 \pm 0.015$ & 1.64 & $9.54 \pm 0.52$ & $8.7_{-1.4}^{+1.6}$ \\
\hline$I-[3.6]>5.0$ & {$[3.6]<17.5$} & $\mathrm{SF}$ & 5683 & 37 & 1.16 & 1.80 & $0.150 \pm 0.012$ & 6.93 & $7.62 \pm 0.61$ & $4.3_{-0.9}^{+1.2}$ \\
\hline$R-K_{s}>5.0$ & $K_{s}<17.9$ & $\mathrm{E}$ & 1761 & 82 & 1.04 & 1.80 & $0.437 \pm 0.038$ & 3.52 & $13.17 \pm 1.15$ & $23.5_{-5.8}^{+6.7}$ \\
\hline$R-K_{s}>5.0$ & $K_{s}<17.9$ & SF & 394 & 18 & 1.06 & $\ldots$ & $\ldots$ & $\ldots$ & $\ldots$ & $\ldots$ \\
\hline$R-K_{s}>5.0$ & $K_{s}<18.4$ & $\mathrm{E}$ & 3804 & 71 & 1.08 & 1.80 & $0.354 \pm 0.024$ & 3.13 & $11.86 \pm 0.80$ & $17.0_{-3.3}^{+3.8}$ \\
\hline$R-K_{s}>5.0$ & $K_{s}<18.4$ & $\mathrm{SF}$ & 1591 & 29 & 1.11 & 1.80 & $0.273 \pm 0.032$ & 3.10 & $10.47 \pm 1.23$ & $11.6_{-3.7}^{+4.7}$ \\
\hline$I-K_{s}>4.0$ & $K_{s}<17.9$ & $\mathrm{E}$ & 802 & 85 & 1.07 & 1.80 & $0.342 \pm 0.056$ & 2.03 & $12.69 \pm 2.08$ & $21.0_{-8.9}^{+12.2}$ \\
\hline$I-K_{s}>4.0$ & $K_{s}<17.9$ & SF & 138 & 15 & 1.15 & $\ldots$ & $\ldots$ & $\ldots$ & $\ldots$ & $\ldots$ \\
\hline$I-K_{s}>4.0$ & $K_{s}<18.4$ & $\mathrm{E}$ & 1810 & 68 & 1.13 & 1.80 & $0.423 \pm 0.037$ & 3.66 & $13.44 \pm 1.18$ & $25.1_{-6.2}^{+7.4}$ \\
\hline$I-K_{s}>4.0$ & $K_{s}<18.4$ & $\mathrm{SF}$ & 843 & 32 & 1.18 & 1.80 & $0.364 \pm 0.055$ & 2.87 & $11.98 \pm 1.81$ & $17.6_{-7.0}^{+9.6}$ \\
\hline
\end{tabular}

Notes. Clustering results for samples with $N<500$ are not shown due to poor-quality statistics for our field area. The ERO types are labeled as E for passive EROs and SF for star-forming EROs. \% total is the fraction of combined EROs in a particular ERO-type sub-population. These samples contain only objects that have $z_{\text {phot }}$ information. 


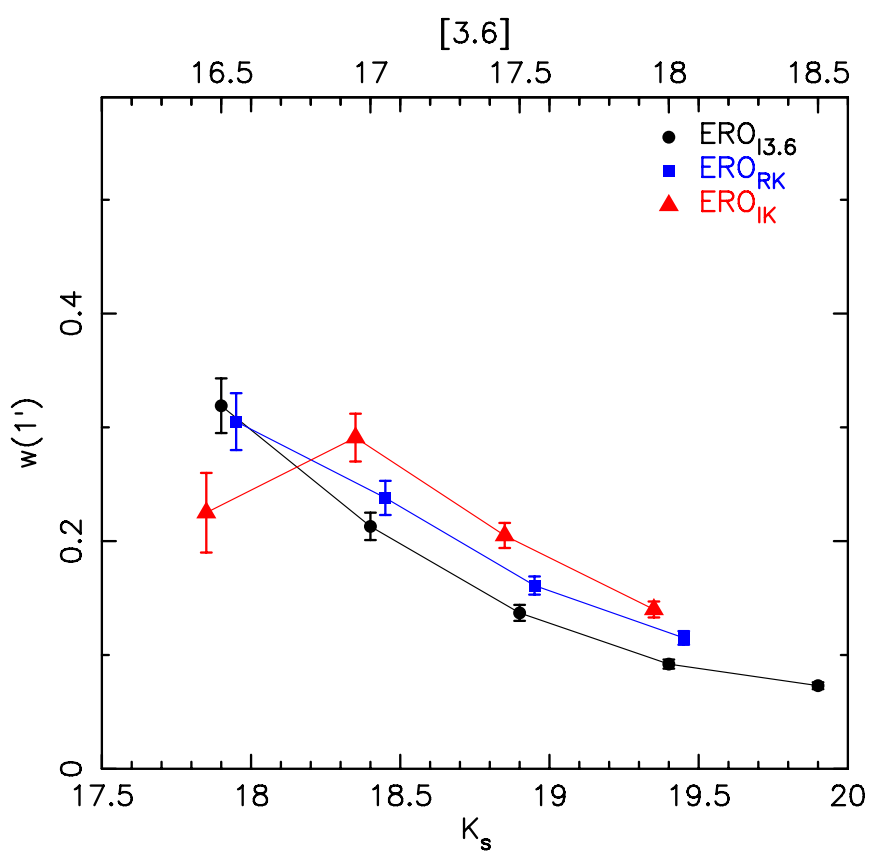

Figure 12. Angular correlation strength of our ERO samples as a function of apparent magnitude and for a fixed slope of $\gamma=1.80$. For clarity, magnitude offsets of +0.05 and -0.05 were applied to the $\mathrm{ERO}_{I 3.6}$ and $\mathrm{ERO}_{I K}$ data, respectively. Angular clustering is seen to be strongly dependent on sample limiting magnitude. Here we have used $K_{s} \approx[3.6]+1.4$, based on the average of $K_{s}-$ [3.6] for our EROs, to place the [3.6] samples on the figure.

(A color version of this figure is available in the online journal.)

for our EROs). A complete summary of our combined ERO angular clustering analysis results is presented in Tables 1 and 3. For ERO samples sub-divided by type, we observe stronger angular clustering for passive EROs in all three ERO samples. In all cases but the $\mathrm{ERO}_{R K} K_{s}<17.9$ sample, the $w\left(1^{\prime}\right)$ clustering amplitude of the star-forming and passive ERO sub-samples straddles that of the combined ERO sample. Figure 13 shows angular correlation functions for $\mathrm{ERO}_{I 3.6}$ [3.6] < 17.5 combined and type-separated samples. There is no clear distinction between the passive, star-forming, and mixed
$\mathrm{ERO}_{R K}$ and $\mathrm{ERO}_{I K}$ samples, but the trend is the same as for the $\mathrm{ERO}_{I 3.6}$ sample. Separated sample results are presented in Tables 2 and 4.

Freely fitted values for $\gamma$ were found to range between $\gamma=1.58$ and 1.98 for the combined ERO samples with a typical uncertainty of $\sigma_{\gamma}=0.05-0.10$. The passive EROs consistently yield a steeper power-law index than the star-forming ERO populations (excepting the $\mathrm{ERO}_{R K} K_{S}<17.9$ sample), as can clearly be seen in Figure 13. If we take the slope of $\gamma$ as an indicator of environment, where on the scale of $\approx 1$ Mpc a steep slope implies a more clustered distribution and a shallow $\gamma$ a smoother distribution, this implies that passive EROs typically occupy denser environments than star-forming EROs.

\subsection{ERO Redshift Distribution}

To transform the angular correlation function to a real-space correlation function, we need to know the distribution of EROs in redshift. The redshift distribution (hereafter $d N / d z$ ) for each ERO sample was determined by number counts in bins of $\Delta z_{\text {phot }}=0.02$ over $0<z_{\text {phot }}<2$. The ANN $z$ code assigns galaxies with the same observed photometry to a particular $z_{\text {phot }}$, while the true $z_{\text {spec }}$ for these galaxies will be scattered above and below the $z_{\text {phot }}$ and in some cases catastrophically under/overestimated (see Figure 2). In our particular case, since the ERO cut selects a particular redshift distribution analogous to a redshift bin, we expect the true redshift distribution to be somewhat broader than given by the $d N / d z\left(z_{\text {phot }}\right)$. This effect is clearly observed in Figure 6. We therefore need to model the true $d N / d z(z)$ based on the $d N / d z\left(z_{\text {phot }}\right)$.

We build our $d N / d z(z)$ models in a two-stage process. First, we replace the $z_{\text {phot }}$ of each galaxy with a Gaussian probability distribution accounting for $90 \%$ of each galaxy's distribution in redshift, where $\mu=z_{\text {phot }}$ and $\sigma$ is a function of $I$-band magnitude, $\left(B_{w}-R\right)$ color, and $z_{\text {phot }}$. This accounts for the accuracy of the photometric redshift itself. Second, we account for catastrophic $z_{\text {phot }}$ errors with a second Gaussian probability distribution making up the remaining $10 \%$ of each galaxy's distribution in redshift, where $\mu=z_{\text {phot }}$ and $\sigma=0.5$. This corresponds to the $\approx 6 \%$ of galaxies having $\Delta_{z}>3 \sigma_{z} z /(1+z)$, as observed in the data. The outliers need not be precisely modeled,

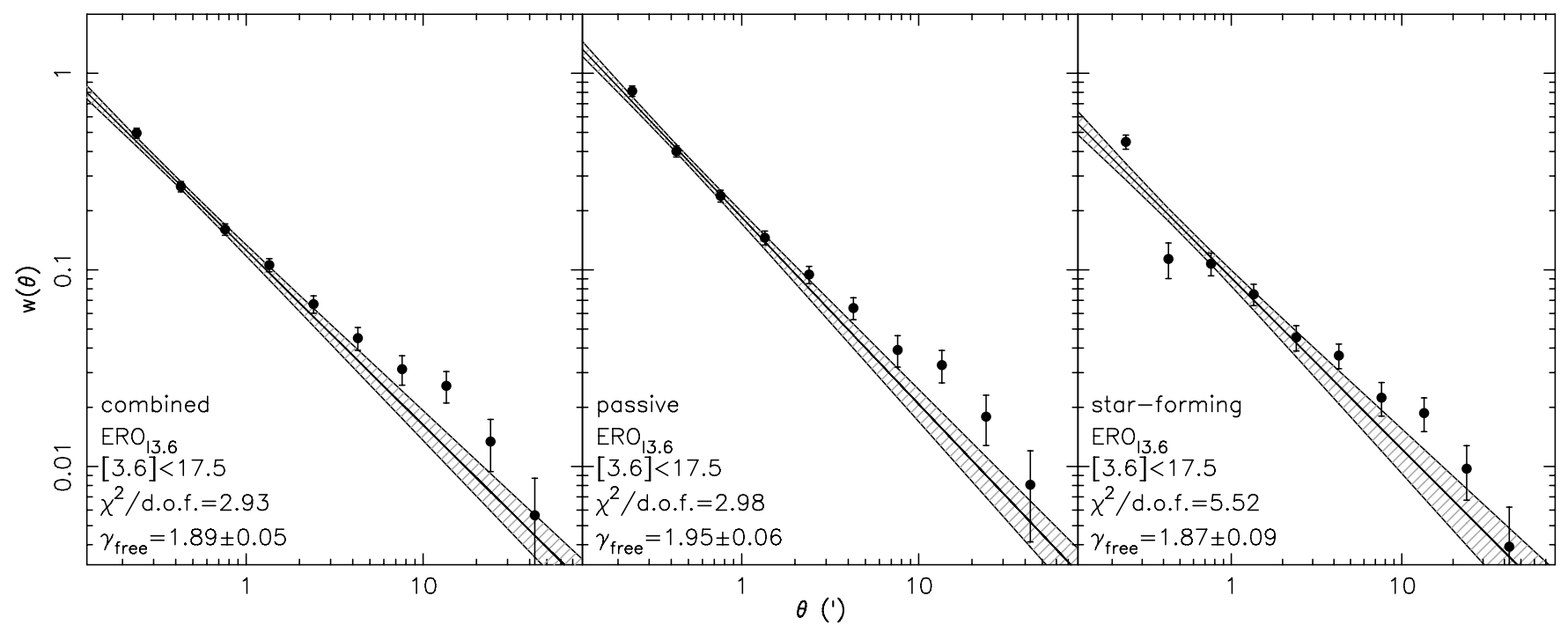

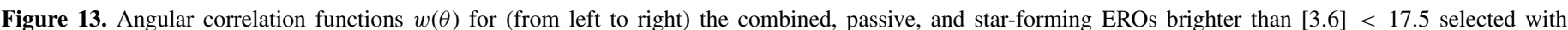

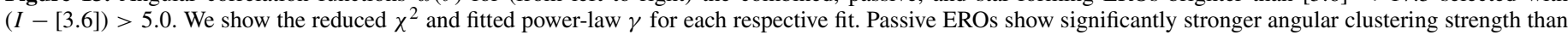
those with star formation, especially on small angular scales. 
as the Limber (1954) equation (see Section 4.3) depends on $(d N / d z)^{2}$. The choice of $10 \%$ was made to better match the $z_{\text {spec }}$ distribution. Our resultant $d N / d z(z)$ models are reasonable representations of the true redshift distribution, as shown by the examples in Figure 6.

The $\bar{z}$ for each of our ERO samples is given in Tables 1-4, and for the reasons discussed in Section 2.2 we do not quote uncertainties on these values. As demonstrated by Figure 6, the $\mathrm{ERO}_{R K}$ sample has lower $\bar{z}$ than both the $\mathrm{ERO}_{I K}$ and $\mathrm{ERO}_{I 3.6}$ samples, while the $\mathrm{ERO}_{I 3.6}$ and $\mathrm{ERO}_{I K}$ have similar $\bar{z}$. Fainter magnitude-limited samples have higher $\bar{z}$, which is expected as a fainter limiting magnitude will include a larger number of more distant galaxies. The passive EROs and star-forming EROs in the type-separated samples have very similar $\bar{z}$ to each other.

Reliable $z_{\text {phot }}$ are only assigned to objects with $I<23.5$; therefore, not all of the EROs in our sample are assigned a $z_{\text {phot }}$. We account for this in two ways. For EROs with no $z_{\text {phot }}$, we assume that they have a $d N / d z(z)$ similar to those with $z_{\text {phot }}$ and simply scale up $d N / d z(z)$ to the full sample size. We do this for completeness, and it has little impact on the clustering results because the Limber (1954) equation (discussed in Section 4.3) only depends on the shape of $d N / d z(z)$. We also provide in Tables 3 and 4 a complete clustering analysis including only objects with $z_{\text {phot }}$ information for comparison. In all but our faintest magnitude-limited samples, $>50 \%$ of EROs in each sample have $z_{\text {phot }}$ information and the modeled $d N / d z$ is considered a valid representation of the entire sample.

It should be noted that when using smaller sample sizes but with an identical $d N / d z(z)$, the clustering length of the

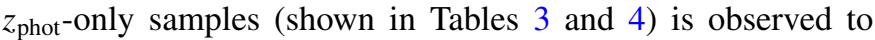
be systematically larger than that of samples including EROs without $z_{\text {phot }}$ information (shown in Tables 1 and 2). For the majority of samples, the increase in $r_{0}$ is $\lesssim 20 \%$, with the exception of samples that have a large number of objects without $z_{\text {phot }}$ information.

We speculate that this is because objects without $z_{\text {phot }}$ information are likely faint and therefore more likely to have a higher average redshift. When included in the angular clustering measurement, these high- $z$ objects increase the number of uncorrelated pairs and will reduce the angular clustering amplitude. This would be accounted for if the redshift distribution was broader to account for the higher redshift of these faint objects. We note that samples with and without complete $z_{\text {phot }}$ information show the same general trends. Since we get better clustering statistics with greater sample size, we use our samples including EROs without $z_{\text {phot }}$ for discussion, and the samples using only objects with a measured $z_{\text {phot }}$ are included as a cross-check.

\subsection{Spatial Clustering}

The spatial correlation function $\xi(\mathbf{r})$ is related to $w(\theta)$ by the Limber (1954) equation,

$$
\begin{aligned}
w(\theta)= & \int_{0}^{\infty} \frac{d N}{d z}\left[\int_{0}^{\infty} \xi\left(\mathbf{r}\left(\theta, z, z^{\prime}\right), z\right) \frac{d N}{d z^{\prime}} d z^{\prime}\right] d z \\
& /\left(\int_{0}^{\infty} \frac{d N}{d z} d z\right)^{2} .
\end{aligned}
$$

The characteristic clustering length $r_{0}$ gives a measure of the clustering strength and is related to $\xi(\mathbf{r})$ by its functional form

$$
\xi(\mathbf{r})=\left(\frac{\mathbf{r}}{r_{0}}\right)^{-\gamma},
$$

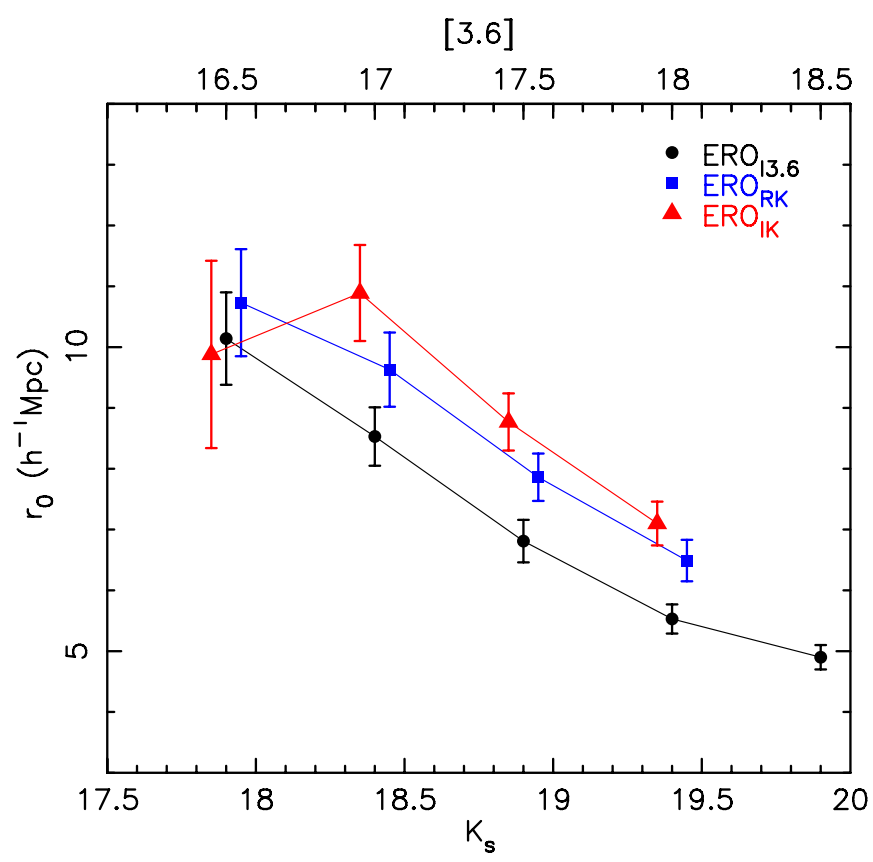

Figure 14. Real-space spatial clustering strength of EROs as a function of apparent magnitude with fixed $\gamma=1.80$ for the three ERO samples. For clarity, magnitude offsets of +0.05 and -0.05 have been applied to the $\mathrm{ERO}_{I 3.6}$ and $\mathrm{ERO}_{I K}$ data, respectively. The spatial clustering is strongly dependent on sample limiting magnitude. Here, we assume $K_{s} \approx$ [3.6] +1.4, based on the average of $K_{s}-$ [3.6] color of our EROs.

(A color version of this figure is available in the online journal.)

where $r_{0}$ is a measure of the spatial separation at which we expect to find an excess pair of galaxies above a random galaxy distribution and $\gamma$ is as previously measured from the angular correlation function. The results of our spatial clustering analysis are given in Tables 1 and 3 for the combined ERO samples and in Tables 2 and 4 for the separated ERO samples. The uncertainty in $r_{0}$ was determined from the spread in $r_{0}$ values of the angular correlation function models with $\chi^{2} \leqslant\left(\chi_{\min }^{2}+1\right)$.

Using our magnitude-limited samples, we explore the spatial clustering of EROs as a function of limiting luminosity. For clarity and ease of comparison to previous studies we will only discuss results using fixed $\gamma=1.80$. In Figure 14 it is clear that ERO clustering strength is a strong function of apparent magnitude, since EROs occupy a particular redshift range, and magnitude is a rough proxy for stellar mass. The $\mathrm{ERO}_{R K}$ and $\mathrm{ERO}_{I K}$ samples show stronger clustering than the $\mathrm{ERO}_{I 3.6}$ samples at similar magnitude limits (as before, we take $K_{s} \approx$ [3.6] +1.4 , based on the average of $K_{s}-$ [3.6] for our EROs). This could be due to the $\mathrm{ERO}_{I 3.6}$ sample having a larger star-forming galaxy fraction.

For the $\mathrm{ERO}_{R K}$ and $\mathrm{ERO}_{I K}$ samples at a fixed magnitude limit, the clustering strength depends on the $\bar{z}$ of each sample, in agreement with previous work (e.g., Kim et al. 2011). This difference is easily accounted for because the difference in $\bar{z}$ between these two samples makes the objects in the $\mathrm{ERO}_{I K}$ sample on average $\approx 6 \%$ brighter for a given limiting magnitude than the $\mathrm{ERO}_{R K}$ sample. With this in mind we speculate that there is very little evolution in $r_{0}$ for roughly equivalent mass EROs between $z=1.2$ and $z=1.0$. The difference between the $\mathrm{ERO}_{I 3.6}$ sample and the $\mathrm{ERO}_{R K}$ and $\mathrm{ERO}_{I K}$ samples we attribute (as above) to the difference in relative star-forming to passive ERO fractions. 
The brightest $\mathrm{ERO}_{I 3.6}, \mathrm{ERO}_{R K}$, and $\mathrm{ERO}_{I K}$ EROs have clustering lengths of $r_{0}=10.14 \pm 0.76 h^{-1} \mathrm{Mpc}, r_{0}=10.73 \pm$ $0.88 h^{-1} \mathrm{Mpc}$, and $r_{0}=9.88 \pm 1.54 h^{-1} \mathrm{Mpc}$, respectively, comparable to that of $\approx 4 L^{*}$ local ellipticals (Sloan Digital Sky Survey, SDSS; e.g., Zehavi et al. 2010). The faintest of $\mathrm{ERO}_{R K}$ and $E R O_{I K}$ samples are still strongly clustered, with $r_{0}=6.49 \pm$ $0.34 h^{-1} \mathrm{Mpc}$ and $r_{0}=7.10 \pm 0.36 h^{-1} \mathrm{Mpc}$, respectively. This is comparable to that of $2 L^{*}$ local ellipticals. With a significantly higher star-forming fraction, the faintest $\mathrm{ERO}_{I 3.6}$ sample shows $r_{0}=4.90 \pm 0.20 h^{-1} \mathrm{Mpc}$, similar to $\approx L^{*}$ local galaxies (SDSS; e.g., Zehavi et al. 2010). It is clear that a larger star-forming fraction leads to reduced clustering strength. Since the star-forming fraction increases with apparent magnitude, this likely contributes to the observed decline of the combined ERO sample clustering length with apparent magnitude seen in Figure 14.

The strong clustering of the faint $\mathrm{ERO}_{R K}$ and $\mathrm{ERO}_{I K}$ samples with respect to the faint $\mathrm{ERO}_{I 3.6}$ samples suggests that there is a dependence of clustering strength on ERO type. The clustering results for our separated $\mathrm{ERO}_{R K}$ and $\mathrm{ERO}_{I K} K_{s}<17.9$ samples are largely inconclusive due to statistical uncertainties. However, all the type-separated ERO samples show a difference between the clustering of passive and star-forming EROs. In the $\mathrm{ERO}_{\text {I3.6 }}$ samples, which have the smallest uncertainties, there is a stark difference in the clustering between the two ERO types, with the clustering length $r_{0}$ of the passive EROs being a factor of $\approx 1.4$ greater than that for the star-forming EROs at all three magnitude limits. It is clear that star-forming EROs occupy very different environments from passive EROs with comparable apparent magnitudes, and this is discussed further in Section 4.4 .

As a cross-check for the reliability of our ERO separation technique (Section 3.2), we tested in the $\mathrm{ERO}_{I 3.6}$ sample out to [3.6] $<17.5$, the clustering of the $944924 \mu \mathrm{m}$ non-detections. For a fixed $\gamma=1.80$ we estimate $r_{0}=$ $8.46 \pm 0.48 h^{-1} \mathrm{Mpc}$, and this agrees well with the clustering length of the corresponding passive sample. We then tested the clustering of the 12,344 $24 \mu \mathrm{m}$ detections for a fixed $\gamma=1.80$ and estimate $r_{0}=6.22 \pm 0.40 \mathrm{~h}^{-1} \mathrm{Mpc}$, which is similarly in agreement with the clustering length of the corresponding star-forming sample. Both results are slightly higher than for the color separated EROs. We take this as evidence that color classified passive EROs with $24 \mu \mathrm{m}$ detections increase the measured clustering length of the sample with $24 \mu$ m detections, while star-forming EROs are absent in the sample with no $24 \mu \mathrm{m}$ detections, and hence there is less likelihood of contamination. This conclusion is supported by the majority of color classified passive EROs with $24 \mu \mathrm{m}$ detections having strong D4000, as seen in Figures 7 and 8. This confirms ERO-type definitions based on $24 \mu \mathrm{m}$ colors as a robust method for separating starforming and passive EROs.

\subsection{Linking EROs throughout z via Spatial Clustering}

The clustering of passive and star-forming EROs with comparable apparent magnitude shows that the two ERO subpopulations occupy very different environments. This suggests that these sub-populations of EROs are the progenitors of different local universe galaxies. In order to make links between EROs and galaxy populations at other epochs, we present comparisons of clustering for passive and star-forming [3.6] $<16.5$ EROs with samples of some of the largest galaxy clustering studies over $0<z<2.5$.

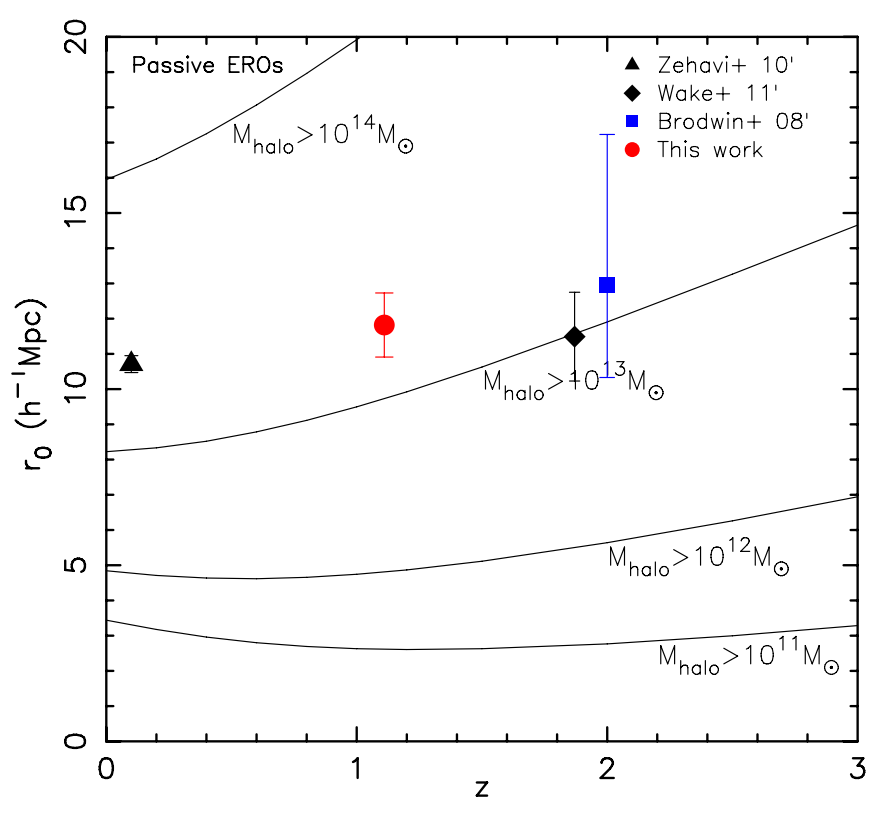

Figure 15. Spatial clustering as a function of redshift for the passive $\mathrm{ERO}_{I 3.6}$ [3.6] < 16.5 sample (red circle; with fixed $\gamma$ for ease of comparison). The Zehavi et al. (2010), $M_{r}^{\max }=-22.0$ sample is indicated by a triangle; the Wake et al. (2011), stellar mass of $=10^{11} M_{\odot}$ sample with a diamond; and the most luminous Brodwin et al. (2008) dust-obscured galaxy sample by the blue square. The curves show the evolution of $r_{0}(z)$ for fixed minimum halo mass, based on the methodology discussed in Section 4.5.

(A color version of this figure is available in the online journal.)

For $\mathrm{ERO}_{\text {I3.6 }}[3.6]<16.5$ passive EROs we use the very brightest samples from each available comparison study and place our results in this context in Figure 15. We have also determined the expected $r_{0}(z)$ for dark matter halo samples and a range of minimum halo masses based on the methodology outlined in Section 4.5. The clustering at fixed minimum halo mass declines with redshift for massive halos (e.g., $M_{\text {halo }}>$ $\left.10^{13} M_{\odot}\right)$. Halo masses, however, grow with time, and we would expect $r_{0}$ for a single, massive, growing halo to be slightly increasing with decreasing redshift. This is also the case for the massive galaxies at their cores. Figure 15 shows that reasonably bright passive EROs are the likely progenitors of $\gtrsim 4 L^{*}$ local universe ellipticals (Zehavi et al. 2010). This suggests that bright EROs are the likely descendants of luminous dust-obscured galaxies (DOGs) at $z \approx 2$ (Brodwin et al. 2008) and the likely progenitors of some of the most massive galaxies in the local universe (Zehavi et al. 2010).

In the case of our $\mathrm{ERO}_{I 3.6}[3.6]<16.5$ star-forming EROs, their clustering strength of $r_{0}=8.89 \pm 1.73 \mathrm{~h}^{-1} \mathrm{Mpc}$ associates them with $\gtrsim 2 L^{*}$ local red galaxies (e.g., Zehavi et al. 2010). Furthermore, when placed into context with comparison studies (Figure 16), the expected evolution in clustering for the $\mathrm{ERO}_{I 3.6}$ [3.6] $<16.5$ star-forming EROs makes these likely progenitors of $z<0.8$ passive galaxies. Furthermore, the clustering length of [3.6] $<16.5$ star-forming EROs is comparable to that of passive [3.6] $<17.5$ EROs. This indicates the possibility of a duty cycle, whereby star-forming EROs turn into passive EROs with significantly fainter apparent magnitudes, which builds a consistent picture. This is evidence that $\gtrsim L^{*}$ local red galaxies are building up stellar mass at $z \approx 1.2$. The $24 \mu \mathrm{m}$ flux limit of MAGES is consistent with that of luminous infrared galaxy emission at $z \approx 1.2$ (Le Floc'h et al. 2005), which sets the minimum SFR at $\approx 10 M_{\odot}$ year $^{-1}$ (Noeske et al. 2007) for star-forming EROs with [3.6] $<17.5$. These galaxies may be 


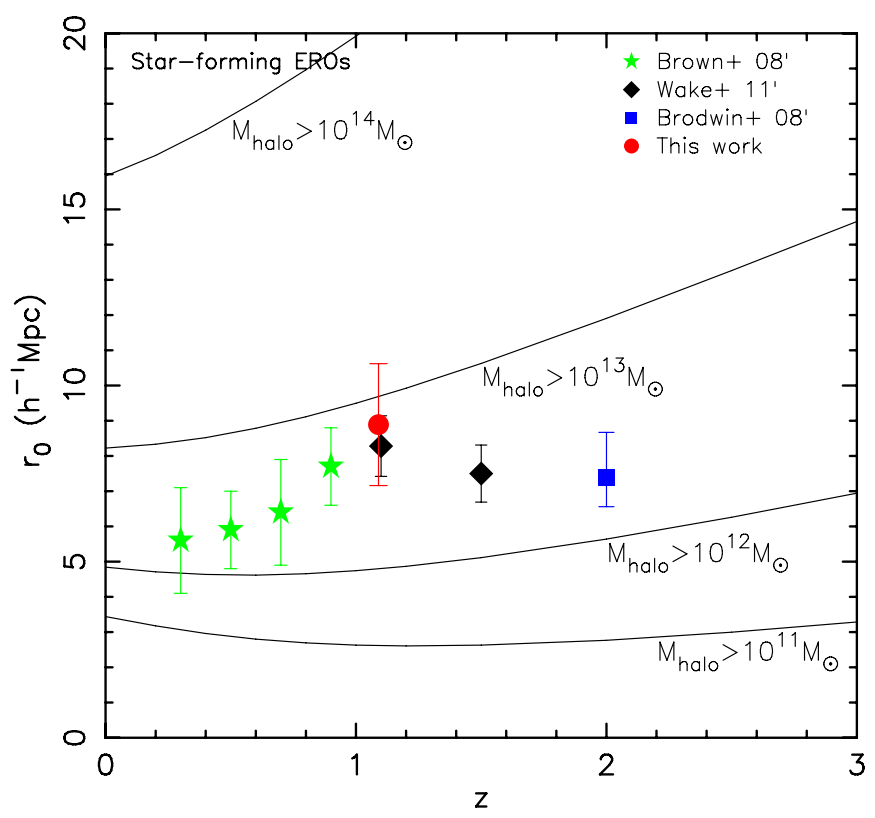

Figure 16. Spatial clustering as a function of redshift for the star-forming ERO $_{I 3.6}[3.6]<17.5$ sample (red circle; with fixed $\gamma$ for ease of comparison). Here, we compare to the brightest absolute magnitude-limited samples of passive red galaxies from Brown et al. (2008), for each redshift range (green stars). The most massive, stellar mass samples from Wake et al. (2011), for each redshift range (diamonds), and the least luminous dust-obscured galaxy sample from Brodwin et al. (2008) (blue square). The curves show the evolution of $r_{0}(z)$ for fixed minimum halo mass, based on the methodology discussed in Section 4.5. (A color version of this figure is available in the online journal.)

undergoing merger-induced star formation or are early forms of present-day ellipticals with continuing/residual star formation.

The above associations provide a strong indication that the ERO population is an earlier form of the elliptical population we observe in the local universe. In general, the $r_{0}$ values for star-forming and passive EROs straddle that of the overall ERO population of the same apparent magnitude, with passive EROs being more strongly clustered than star-forming EROs for a particular magnitude limit.

\subsection{Host Halo Mass}

As galaxies are biased tracers of dark matter, it is possible to get an estimate of their host halo mass through a direct comparison of their clustering with that of dark matter halos. We determine the clustering of dark matter halos from the nonlinear dark matter power spectrum. The power spectrum of dark matter is generated using the transfer function of Eisenstein \& $\mathrm{Hu}$ (1998), the halo mass function and bias of Sheth \& Tormen (1999), and the halo model of Seljak (2000). We assume Navarro-Frenk-White dark matter halo profiles (Navarro et al. 1996) with the concentration scalings of Bullock et al. (2001). We adopt a simple halo occupation scheme assuming one galaxy per halo. We assume that the EROs represent the spatial distribution of their host halos on the scales of $\approx \mathrm{Mpc}$ and determine their host halo mass by matching the $r_{0}$ of the EROs with that of mass-limited dark matter halo samples.

This scheme provides crude estimates of the host halo mass, as it neglects the possibility of ERO satellite galaxies, and the correlation function of galaxies is not well described by a power law. A more accurate treatment may be achieved by halo occupation distribution modeling (e.g., Seljak 2000; Peacock \& Smith 2000; Zheng 2004; Zehavi et al. 2004), which we will address in a future paper. We show the corresponding halo mass estimates for combined ERO samples in Tables 1 and 3 and the separated ERO samples in Tables 2 and 4. We find that our brightest EROs are associated with dark matter halos with masses of the order $10^{13} M_{\odot}$. This host halo mass agrees with that predicted from the halo occupation distribution by Gonzalez-Perez et al. (2011). The faintest EROs are associated with dark matter halos of $\approx 10^{12} M_{\odot}$. These halo masses agree with the ERO-local galaxy population associations made using the spatial clustering measurements in the previous section.

\section{DISCUSSION AND SUMMARY}

We present the most statistically robust measurement of ERO clustering to date. The effects of cosmic variance on our results are modest considering that the integral constraint increases $w(\theta)$ by only $\approx 3 \%$ due to our large survey area. Our redshift distribution was derived from photometric redshifts, which are constrained with spectroscopy, convolved with their photometric uncertainties, and account for catastrophic $z_{\text {phot }}$ outliers, making them reliable representations of $d N / d z(z)$. We find clear trends of decreasing clustering strength with apparent magnitude, which roughly translates to stellar mass since the redshifts are similar. Previous studies have hinted at such a relationship, but the samples were too small to draw solid conclusions (e.g., Brown et al. 2005). Our $\mathrm{ERO}_{R K}$ results agree with our previous measurement of ERO clustering in a sub-field of the NDWFS (Brown et al. 2005). We find that our measured $\mathrm{ERO}_{R K}$ and $\mathrm{ERO}_{I K}$ clustering lengths are typically lower than those of the previous largest area ERO studies by Kim et al. (2011) and Kong et al. (2009) but agree within $2 \sigma$. It should be noted that both Kim et al. (2011) and Kong et al. (2009) have likely underestimated their uncertainties, as they are based on Poisson uncertainties and assume a diagonal covariance matrix, so the true level of disagreement is smaller.

We tested whether the difference in ERO clustering results can be explained by the different criteria used to define ERO samples. We have clearly shown that the various ERO criteria select similar but not identical galaxy populations. Differences in number density, $\bar{z}$, and star-forming to passive ERO fraction are likely the main causes for differing estimates of $r_{0}$ between selection criteria. The results of ERO clustering studies using different selection criteria should be directly comparable so long as these factors are considered. For example, ERO $_{I K} K_{S}<18.4$ EROs possess slightly stronger clustering properties to $\mathrm{ERO}_{R K}$ $K_{s}<18.4$ EROs, because the difference in $\bar{z}$ between the samples means that the $\mathrm{ERO}_{I K}$ EROs are systematically brighter than the $\mathrm{ERO}_{R K}$ EROs. In the case of $\mathrm{ERO}_{I 3.6}$ we found that this criterion selected most of the same galaxies as the $\mathrm{ERO}_{R K}$ and $\mathrm{ERO}_{I K}$ samples, but also a greater surface density of objects for a comparable magnitude. The additional objects included a larger fraction of star-forming EROs than for the $\mathrm{ERO}_{R K}$ and $E \mathrm{O}_{I K}$ samples, and this sample then showed systematically lower clustering for similar magnitude limits. This means that when you are trying to link EROs to local galaxy populations by their clustering, you cannot obtain meaningful results unless you separate the passive and star-forming EROs into separate samples.

Our separation criteria, based on the work of Stern et al. (2006), show strong separation in both $\left(K_{s}-[24]\right)$ and ([3.6] [24]) colors between EROs with star formation and those without. The success of this technique is its ability to pick out PAH emission from hot dust at high redshift and over the redshift range required for ERO samples (Stern et al. 2006; Treyer et al. 
2010). The ([3.6] - [8.0]) color is less effective because $8.0 \mu \mathrm{m}$ is no longer sampling strong PAH features at $z \gtrsim 1$. Likewise the $(J-K)$ color is not useful for most EROs, because it does not bracket or straddle the $4000 \AA$ break until $z \gtrsim 1.4$. Deeper $24 \mu \mathrm{m}$ data would allow this technique to be used for fainter samples than analyzed in this work.

At fixed apparent magnitude, we find that the two ERO sub-populations occupy different environments, with passive EROs being more strongly clustered than those with star formation. The clustering strength for each type-separated ERO sample is also a function of apparent magnitude. In many cases, passive EROs have similar clustering properties to star-forming EROs of brighter apparent magnitude. This is consistent with a duty cycle, whereby star-forming EROs turn into passive EROs with significantly fainter apparent magnitudes.

The SSFR seems to be lower in dense environments at low redshift (e.g., Zehavi et al. 2010), but some studies suggest that this trend changes at $z \gtrsim 1.3$ (e.g., Cooper et al. 2007) to $z \approx 2$ (e.g., Brodwin et al. 2008; Magliocchetti et al. 2008). While we observe that EROs without star formation typically occupy denser environments than star-forming EROs, we see evidence for star formation in much denser environments than observed at $z<1$. This suggests that a transition between overdensities being dominated by passive galaxies to being dominated by star-forming galaxies could well be occurring at $z \gtrsim 1.3$, in agreement with the findings of Cooper et al. (2007) and Brodwin et al. (2008).

Denser environments imply higher mass dark matter halos, and star-forming EROs seem to have host halo masses of up to $M_{\text {halo }} \approx 10^{13} M_{\odot}$, an order of magnitude greater than the host halo mass of a typical star-forming galaxy at $z=0$ (Zheng et al. 2007). The shifting of the peak in star formation to higher mass halos at $z>1$ is in agreement with the results of previous highredshift clustering studies (e.g., Magliocchetti et al. 2008; Wake et al. 2011). This could be evidence for "downsizing," where the host halo mass and stellar mass of star-forming galaxies decrease with decreasing redshift. Since a fixed halo mass at high redshift is more biased with respect to the underlying matter distribution (e.g., Sheth \& Tormen 1999; Seljak 2000) and therefore has a greater merger and mass accretion rate than at $z<1$, this result is not unexpected.

The most significant result of our study is that the passive and star-forming ERO sub-populations are two distinct galaxy populations. Separating the ERO population into its sub-populations has allowed us to place EROs into an evolutionary sequence. Bright passive EROs are the likely progenitors of local $\gtrsim 4 L^{*}$ red galaxies, indicating that massive red galaxies have had their star formation truncated prior to $z \approx 1.2$. We were not able to obtain a clustering measurement for the progenitors of central cluster galaxies as such structures are rare, and as a consequence there are too few of these galaxies to measure their clustering. However, our brightest ERO samples certainly hint that the very brightest EROs are the likely progenitors of the most massive ellipticals in the local universe.

Interestingly, the clustering of star-forming EROs connects them as the likely progenitors of local $\gtrsim L^{*}$ red galaxies. It may seem peculiar that we detect significant star formation $\left(\gtrsim 10 M_{\odot} \mathrm{yr}^{-1}\right.$; Noeske et al. 2007) at $z \approx 1.2$ in progenitors of local $\gtrsim L^{*}$ ellipticals, since it is well established that star formation is expected to have ceased in these objects at $z \approx 1.5$ (e.g., gravitational lensing; Rusin \& Kochanek 2005). However, red/elliptical galaxies can be modeled with tau models with e-folding times of $\tau \lesssim 1$ Gyr and formation redshifts of $z \gtrsim 2$.
It is thus possible to have a galaxy where the bulk of star formation took place at $z \gtrsim 1.5$, but at $z=1.2$ the SFR is still $\approx 10 M_{\odot} \mathrm{yr}^{-1}$ while by $z=0.5$ the SFR is negligible. Therefore, we find that local red galaxies, down to $L^{*}$, in some cases were still building stellar mass via star formation at this epoch.

This work is based in part on observations made with the Spitzer Space Telescope, Spitzer/IRAC, and Spitzer/MIPS, which is operated by the Jet Propulsion Laboratory, California Institute of Technology, under a contract with NASA. This work is based in part on observations made with the Kitt Peak National Observatory (KPNO). This research was supported by the National Optical Astronomy Observatory, which is operated by the Association of Universities for Research in Astronomy (AURA), Inc., under a cooperative agreement with the National Science Foundation. We thank our colleagues on the NDWFS, SDWFS, and MAGES teams. We thank Renbin Yan for providing the DEEP2 EGS spectroscopic data used in this work, Mark Dickinson for providing the FIDEL EGS catalog used in this work, $\mathrm{Xu}$ Kong for providing the COSMOS ERO surface density data from Kong et al. (2009), and Chris Conselice for providing the DEEP2 ERO surface density data from Conselice et al. (2008). D.P.P. acknowledges support from an Australian postgraduate award (APA) and a J.L. William postgraduate award. M. J. I. B. acknowledges support from an Australian Research Council (ARC) Future Fellowship.

\section{REFERENCES}

Abadi, M. G., Lambas, D. G., \& Muriel, H. 1998, ApJ, 507, 526 Ashby, M. L. N., Stern, D., Brodwin, M., et al. 2009, ApJ, 701, 428 Barmby, P., Huang, J., Ashby, M. L. N., et al. 2008, ApJS, 177, 431 Bertin, E., \& Arnouts, S. 1996, A\&AS, 117, 393

Brodwin, M., Brown, M. J. I., Ashby, M. L. N., et al. 2006, ApJ, 651, 791 Brodwin, M., Dey, A., Brown, M. J. I., et al. 2008, ApJL, 687, 65 Brown, M. J. I., Dey, A., Jannuzi, B. T., et al. 2007, ApJ, 654, 858 Brown, M. J. I., Jannuzi, B. T., Dey, A., \& Tiede, G. P. 2005, ApJ, 621, 41 Brown, M. J. I., Zheng, Z., White, M., et al. 2008, ApJ, 682, 937 Bullock, J. S., Kolatt, T. S., Sigad, Y., et al. 2001, MNRAS, 321, 559 Bundy, K., Ellis, R. S., Conselice, C. J., et al. 2006, ApJ, 651, 120 Cimatti, A., Daddi, E., Mignoli, M., et al. 2002, A\&A, 381, 68 Conselice, C. J., Bundy, K., U, V., et al. 2008, MNRAS, 383, 1366 Cooper, M. C., Newman, J. A., Coil, A. L., et al. 2007, MNRAS, 376, 1445 Daddi, E., Cimatti, A., Broadhurst, T., et al. 2002, A\&A, 384, L1 Daddi, E., Cimatti, A., Pozzetti, L., et al. 2000, A\&A, 361, 535 Dey, A., Graham, J. R., Ivison, R. J., et al. 1999, ApJ, 519, 610 Dey, A., Willmer, C., Papovich, C., et al. 2007, NOAO Proposal ID \#2007A0147, 147

Dickinson, M., \& FIDEL team., 2007, BAAS, 38, 822

Eisenstein, D. J., \& Hu, W. 1998, ApJ, 496, 605

Elston, R., Rieke, G. H., \& Rieke, M. J. 1988, ApJL, 331, 77

Firth, A. E., Lahav, O., \& Somerville, R. S. 2003, MNRAS, 339, 1195

Firth, A. E., Somerville, R. S., McMahon, R. G., et al. 2002, MNRAS, 332, 617 Georgakakis, A., Afonso, J., Hopkins, A. M., et al. 2005, ApJ, 620, 584

Gonzalez-Perez, V., Baugh, C. M., Lacey, C. G., \& Almeida, C. 2009, MNRAS, 398, 497

Gonzalez-Perez, V., Baugh, C. M., Lacey, C. G., \& Kim, J.-W. 2011, MNRAS, 417,517

Graham, J. R., \& Dey, A. 1996, ApJ, 471, 720

Groth, E. J., \& Peebles, P. J. E. 1977, ApJ, 217, 385

Jannuzi, B., Weiner, B., Block, M., et al. 2010, BAAS, 42, 513

Jannuzi, B. T., \& Dey, A. 1999, in ASP Conf. Ser. 191, Photometric Redshifts and the Detection of High Redshift Galaxies, ed. R. Weymann, L. StorrieLombardi, M. Sawicki, \& R. Brunner (San Francisco, CA: ASP), 111

Kim, J., Edge, A. C., Wake, D. A., \& Stott, J. P. 2011, MNRAS, 410, 241 Kochanek, C. S., Eisenstein, D. J., Cool, R. J., et al. 2012, ApJS, 200, 8 Kong, X., Daddi, E., Arimoto, N., et al. 2006, ApJ, 638, 72

Kong, X., Fang, G., Arimoto, N., \& Wang, M. 2009, ApJ, 702, 1458

Lacy, M., Storrie-Lombardi, L. J., Sajina, A., et al. 2004, ApJS, 154, 166 Landy, S. D., \& Szalay, A. S. 1993, ApJ, 412, 64 
Le Floc'h, E., Papovich, C., Dole, H., et al. 2005, ApJ, 632, 169

Limber, D. N. 1954, ApJ, 119, 655

Magliocchetti, M., Cirasuolo, M., McLure, R. J., et al. 2008, MNRAS, 383, 1131

Messias, H., Afonso, J., Hopkins, A., et al. 2010, ApJ, 719, 790

Moustakas, L. A., Casertano, S., Conselice, C. J., et al. 2004, ApJL, 600, 131

Moustakas, L. A., \& Somerville, R. S. 2002, ApJ, 577, 1

Navarro, J. F., Frenk, C. S., \& White, S. D. M. 1996, ApJ, 462, 563

Newman, J. A., Cooper, M. C., Davis, M., et al. 2012, arXiv:1203.3192

Noeske, K. G., Weiner, B. J., Faber, S. M., et al. 2007, ApJL, 660, 43

Norberg, P., Baugh, C. M., Hawkins, E., et al. 2002, MNRAS, 332,827

Peacock, J. A., \& Smith, R. E. 2000, MNRAS, 318, 1144

Pozzetti, L., \& Mannucci, F. 2000, MNRAS, 317, L17

Roche, N. D., Almaini, O., Dunlop, J., Ivison, R. J., \& Willott, C. J. 2002, MNRAS, 337, 1282

Rusin, D., \& Kochanek, C. S. 2005, ApJ, 623, 666

Seljak, U. 2000, MNRAS, 318, 203
Sheth, R. K., \& Tormen, G. 1999, MNRAS, 308, 119

Smail, I., Owen, F. N., Morrison, G. E., et al. 2002, ApJ, 581, 844

Spergel, D. N., Bean, R., Doré, O., et al. 2007, ApJS, 170, 377

Spinrad, H., Dey, A., Stern, D., et al. 1997, ApJ, 484, 581

Springel, V., White, S. D. M., Jenkins, A., et al. 2005, Natur, 435, 629

Stern, D., Chary, R., Eisenhardt, P. R. M., \& Moustakas, L. A. 2006, AJ, 132,1405

Stern, D., Eisenhardt, P., Gorjian, V., et al. 2005, ApJ, 631, 163

Treyer, M., Schiminovich, D., Johnson, B. D., et al. 2010, ApJ, 719, 1191

Wake, D. A., Whitaker, K. E., Labbé, I., et al. 2011, ApJ, 728, 46

Wilson, G., Huang, J.-S., Fazio, G. G., et al. 2007, ApJL, 660, 59

Wilson, G., Huang, J.-S., Pérez-Gonzlez, P. G., et al. 2004, ApJS, 154, 107

Yan, L., \& Thompson, D. 2003, ApJ, 586, 765

Yan, R., Newman, J. A., Faber, S. M., et al. 2009, MNRAS, 398, 735

Zehavi, I., Weinberg, D. H., Zheng, Z., et al. 2004, ApJ, 608, 16

Zehavi, I., Zheng, Z., Weinberg, D. H., et al. 2011, ApJ, 701, 59

Zheng, Z. 2004, ApJ, 610, 61

Zheng, Z., Coil, A. L., \& Zehavi, I. 2007, ApJ, 667, 760 